\title{
Fiber R and D for the CMS HCAL
}

\author{
H. S. Budd, A. Bodek, P. de Barbaro, D. Ruggiero, E. Skup \\ Department of Physics and Astronomy, University of Rochester, Rochester, NY 14627 \\ To be published in the proceeding of SCIFI97 Conference, \\ November 3-6, 1997, Notre Dame, Indiana.
}

\begin{abstract}
.
This paper documents the fiber $\mathrm{R}$ and $\mathrm{D}$ for the CMS hadron barrel calorimeter (HCAL). The R and D includes measurements of fiber flexibility, splicing, mirror reflectivity, relative light yield, attenuation length, radiation effects, absolute light yield, and transverse tile uniformity. Schematics of the hardware for each measurement are shown. These studies are done for different diameters and kinds of multiclad fiber.
\end{abstract}

The CMS HCAL optical design is similar to the CDF Plug Upgrade optical design [1] [2]. A wavelength shifting (WLS) fiber, containing K27 waveshifter, embedded in the tile collects the scintillation light. Outside the tile, the WLS fiber is spliced to a clear fiber. The clear fiber takes the light to a connector at the edge of the pan. An optical cable brings the light to the optical readout box. The readout box assembles the light from layers to towers and brings the light to the photodetectors.

The fibers tested for this paper are all multiclad. The diameters of the fibers range from $0.83 \mathrm{~mm}$ to $1.0 \mathrm{~mm}$. CMS has chosen fiber of $0.94 \mathrm{~mm}$ diameter. We test four types of Kuraray fiber, non-S (S-25), S-35, S-50, and S (S-70) [3]. The most flexible Kuraray fiber is $\mathrm{S}$ type and the least flexible fiber is non-S type. We test two batches of WLS Bicron fiber, BCF91A-MC [4]. Batch 1 is an earlier version than Batch 2. We test one batch of Bicron clear fiber, BCF-98 MultiClad, which was made at the same time at Batch 1 WLS fiber. The waveshifter for all WLS fiber is K27.

We use R580-17 phototubes. This tube is a Hamamatsu 1.5 inch diameter, 10 stage tube with a green extended photocathode. The R580-17 photocathode and the photocathode for the CMS photodetectors, HPDs, are the same.

For most measurements a tile excited by a radioactive source generates the light. This insures that the spectrum of light is the same for these tests as the 
TABLE 1. Flexibility of different fibers. Column 1 gives the kind of fiber. Column 2 gives the smallest bend diameter without the fiber damage. Column 3 gives the largest bend diameter with fiber damage.

\begin{tabular}{|l|c|c|}
\hline Fiber type & Fiber not damaged & Fiber Damaged \\
\hline Kuraray $1 \mathrm{~mm}$ non-S & $21 / 2 \mathrm{in}$ & 2 in \\
Kuraray 0.94 mm S-35 & $3 / 4$ in & $5 / 8$ in \\
Kuraray 0.94 mm S-50 & $5 / 8$ in & - \\
Kuraray 0.83 mm S & $5 / 8$ in & - \\
Bicron $1.00 \mathrm{~mm}$ & 2 in & $11 / 2$ in \\
\hline
\end{tabular}

spectrum from the CMS HCAL calorimeter. We use both a Cs-137 $\gamma$ source and a Ru-106 $\beta$ source. The Cs-137 source is collimated by a lead cone. The widest diameter of the cone is $7.5 \mathrm{~cm}$. A picoammeter reads out the phototube. The data aquisition (DAQ) program averages 20 measurements from the picoammeter and creates a pedestal subtracted data file. The absolute light yield measurement uses the Ru-106 source. Its DAQ consists of a 2249A Lecroy ADC triggered by a coincidence of two scintillation counters.

The optical connectors used for these measurements were developed by DDK [5] and CDF Plug Upgrade Group [6] [7]. Their part numbers are MCP-10P-1 (0.83 mm fiber), MCP-10P-2 (0.90 mm fiber), MCP-10P-3 (1.00 mm fiber), and MCP-10A (the connector housing).

\section{FIBER FLEXIBILITY}

We have studied Kuraray non-S fiber for flexibility by looking at the change in light transmission after the fibers are bent. Fibers with a change in transmission greater than $2 \%$ always have cracks or crazing in the bent portions. They have light leaking out from these cracks. Hence, we test for flexibility by looking for light leaking out of the bend portions.

We test the flexibility by wrapping the fibers around dowels and looking for cracks where light leaks out. Table 1 gives the result. The test lasted $1 / 2$ year. If the fiber develops cracks, the cracks appear $1 / 2-2$ days after the fibers are wrapped around the dowel. The smallest fiber bend diameter for the HCAL barrel is 3 inches. Both the Kuraray S-35 fiber and Bicron fiber are flexible enough for CMS HCAL.

\section{FIBER SPLICING}

Fiber splicing is done with the semi-automated splicer developed by the CDF Plug Upgrade Group [8]. Splice transmission of WLS fibers is measured by scanning across a splice using the CDF automated UV scanner [8], see Figure 1. Figure 2a shows the results of splicing tests. Table 2 lists the 
results of the tests. Only splicing tests done at the same time should be compared. The Dec 96 splicing test shows that non-S fiber splices have higher transmission than $\mathrm{S}$ type fiber. This result confirms CDF's measurement of the difference in splice transmission between non-S and $S$ type fibers [8]. The Nov 97 splicing test shows the splice transmission for non-S and S-35 fiber is the same. Splice transmission for S-50 is worse than non-S and S-35 fiber. We have chosen S-35 fiber for the HCAL preproduction prototype because of its excellent flexibility and high splice transmission. The Sept 96 splicing test shows that the splice transmission for Kuraray non-S fiber and Bicron fiber is the same.

The fiber ends must be polished well for good splice transmission. We have compared splice transmission using two different polishing techniques. One polishing technique uses the Avtech polisher [7]. The Avtech polisher is a single fiber polisher which CDF used for its production. The second technique is ice polishing. Ice polishing was pioneered by Fermilab Charmonium experiment E835 [9], and was used by Fermilab experiment HyperCP E871 [10], and D0. The technique involves freezing fibers in water and polishing the fiber/ice combination. Many fibers can be polished at once with ice polishing. Figure $2 \mathrm{~b}$ shows the results of splicing Kuraray $0.94 \mathrm{~mm}$, S-35 fibers with these two techniques. We conclude that both polishing techniques give the same transmission. CMS HCAL has chosen to ice polish their fibers.

We have tested the splice transmission of clear fibers. Figure 3 shows the setup used to measure the transmission of the splice. A connector and fiber assembly with WLS fiber at the nonconnector end is called a "pigtail". The WLS fibers in a "pigtail" are inserted into a tile and then the tile is excited by a radioactive source to readout the light. The pigtail for this test consists of $20 \mathrm{~cm}$ WLS fibers spliced to $99 \mathrm{~cm}$ clear fibers in a connector. The WLS fiber inserted into a tile injects a constant amount of light into the clear fiber. After the clear fiber is cut and spliced, the pigtail is remeasured. The ratio of the measurement before and after the splice is defined as the splice transmission. The results are shown in Figure 4. The test shows that the splice transmission for clear non-S and clear S-35 fiber is the same. Both clear and WLS S-35 splicing tests are done with the same splicing machine by the same operator. Table 2 lists all the fiber splicing results.

\section{MIRROR REFLECTIVITY}

The ends of the fibers are mirrored using vacuum deposition in Lab 7 at Fermilab. A brief description of the mirroring procedure is given in reference [10]. We have studied the mirror reflectivity for 2 types of fibers and both polishing techniques.

The reflectivity is measured using the automated UV scanner (see Figure 1). A mirrored fiber is put in a connector and measured with the UV scanner near 
FIGURE 1. CDF automated UV scanner.

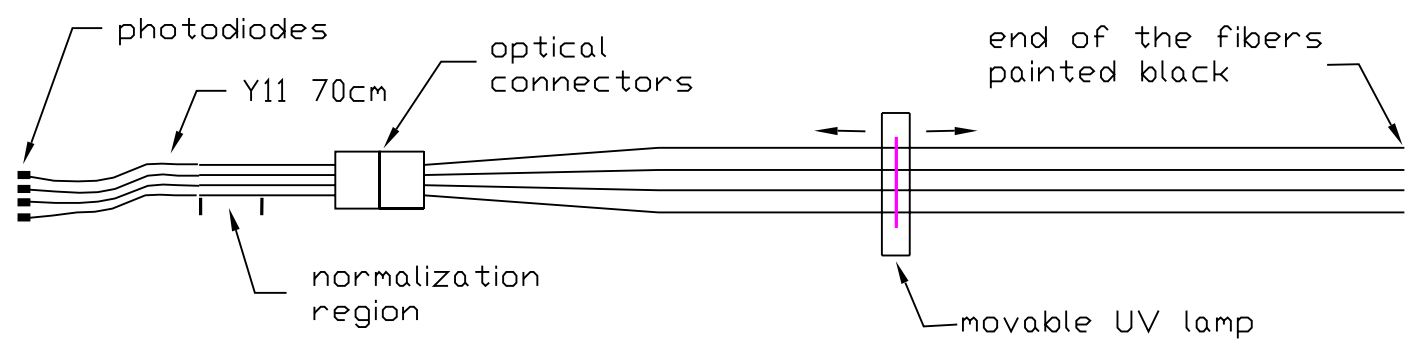

FIGURE 2. Splice transmission for WLS fibers. (a) compares different kinds of fibers and (b) compares two different polishing techniques.
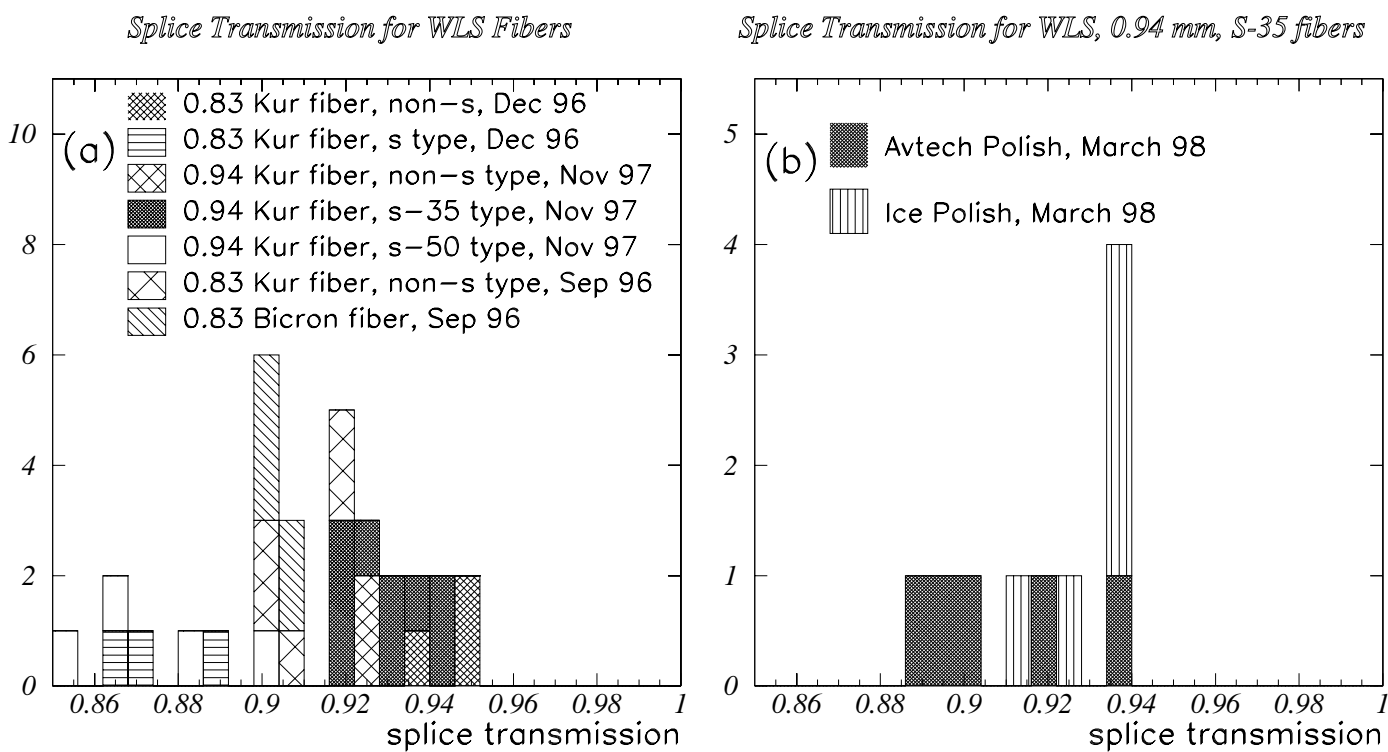

the mirror. Next, the mirror is cut off at $45^{\circ}$ to the fiber axis. The end of the fiber is painted black to prevent any reflection from the end of the fiber. The fiber is remeasured with the automated UV scanner. The reflectivity is defined as (measurement with mirror)/(measurement without mirror) - 1 .

Figure 5 shows the mirror reflectivity results. We have measured the reflectivity of a $31 / 2$ year old CDF pigtail, which was a spare for the CDF End Plug Hadron Calorimeter [7]. The pigtail was made of $0.83 \mathrm{~mm}$, non-S fibers polished with the Avtech polisher. The mirror was dipped in Red Spot UV curable coating to protect the mirror. The measurement shows no degeneration in the mirror after $31 / 2$ years. A measurement of recently mirrored non-S type fiber gives the same reflectivity. The reflectivity of S-35 fibers is roughly $5 \%$ lower. The reflectivity for ice polished fibers seems to be slightly 
TABLE 2. Splice transmission of different kinds of fibers and different polishing techniques. Column 1 gives the kind of fiber. Column 2 gives the fluor. Column 3 gives the kind of polish. Column 4 gives the date of the test. Column 5 gives the number of fibers tested. Column 6 gives the mean of the splice distribution. Column 7 gives the RMS of the distribution. Only tests done on the same date should be compared.

\begin{tabular}{|l|c|c|c|c|c|c|}
\hline Fiber type & Fluor & Polish & Date & Number & Mean & RMS \\
\hline Kuraray 0.83 mm non-S & K27 & Avtech & Dec 96 & 3 & 0.948 & 0.006 \\
Kuraray 0.83 mm S & K27 & Avtech & Dec 96 & 3 & 0.876 & 0.009 \\
Kuraray 0.94 mm non-S & K27 & Avtech & Nov 97 & 2 & 0.928 & - \\
Kuraray 0.94 mm S-35 & K27 & Avtech & Nov 97 & 9 & 0.930 & 0.009 \\
Kuraray 0.94 mm S-50 & K27 & Avtech & Nov 97 & 4 & 0.879 & 0.019 \\
Kuraray 0.83 mm non-S & K27 & Avtech & Sep 96 & 5 & 0.908 & 0.009 \\
Bicron 0.83 mm & K27 & Avtech & Sep 96 & 5 & 0.902 & 0.003 \\
Kuraray 0.94 mm S-35 & K27 & Avtech & Mar 98 & 5 & 0.908 & 0.018 \\
Kuraray 0.94 mm S-35 & K27 & Ice & Mar 98 & 5 & 0.930 & 0.008 \\
Kuraray 0.94 mm non-s & clear & Avtech & Nov 97 & 5 & 0.904 & 0.018 \\
Kuraray 0.94 mm S-35 & clear & Avtech & Nov 97 & 5 & 0.893 & 0.011 \\
\hline
\end{tabular}

FIGURE 3. Setup used to measure transmission of clear to clear splice. Figure 4 shows the results.

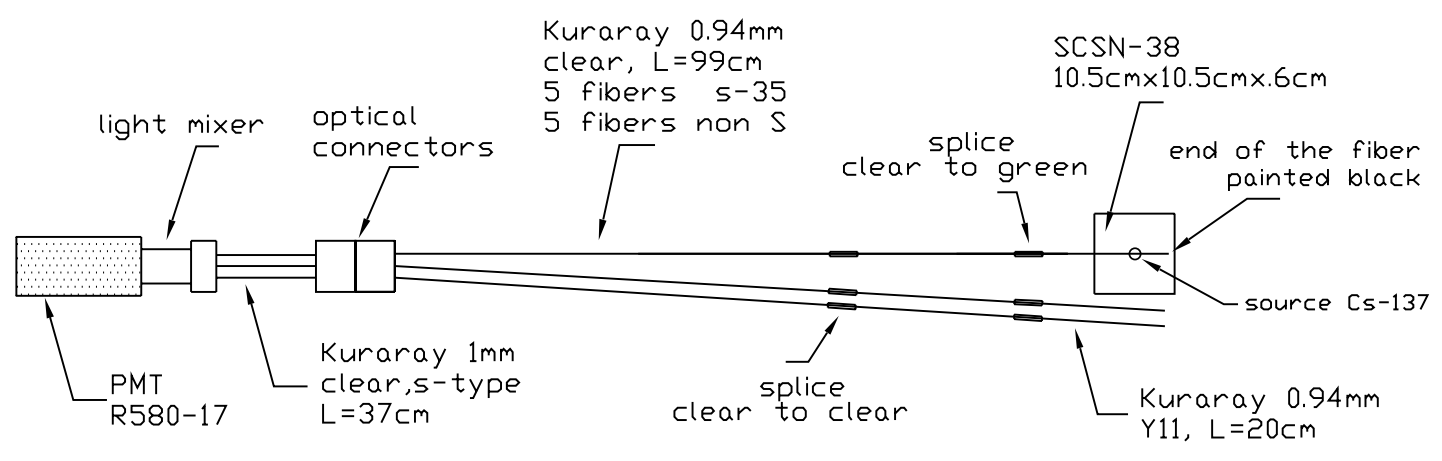

lower than Avtech polished fibers. Figure 5b shows the mirror reflectivity for Kuraray and Bicron fiber are very similar.

We have measured the light increase from the mirror. A pigtail with three fibers, shown in Figure 6, is made and scanned with the UV scanner. The pigtail is also measured with a tile and phototubes as shown in Figure 6. Next, the mirror is cut off at $45^{\circ}$ to the fiber axis, and the end of the fiber is painted black. The pigtail is remeasured with both setups. We get the following:

$$
\frac{\left(\frac{\text { light from tile with mirror }}{\text { light from tile with no mirror }}\right)}{\left(\frac{\text { light from UV scanner with mirror }}{\text { light from UV scanner with no mirror }}\right)}=0.865 \text { with RMS }=0.004
$$

This is used to transfer reflectivity measurements using the UV scanner into 
FIGURE 4. Splice transmission for clear fibers.

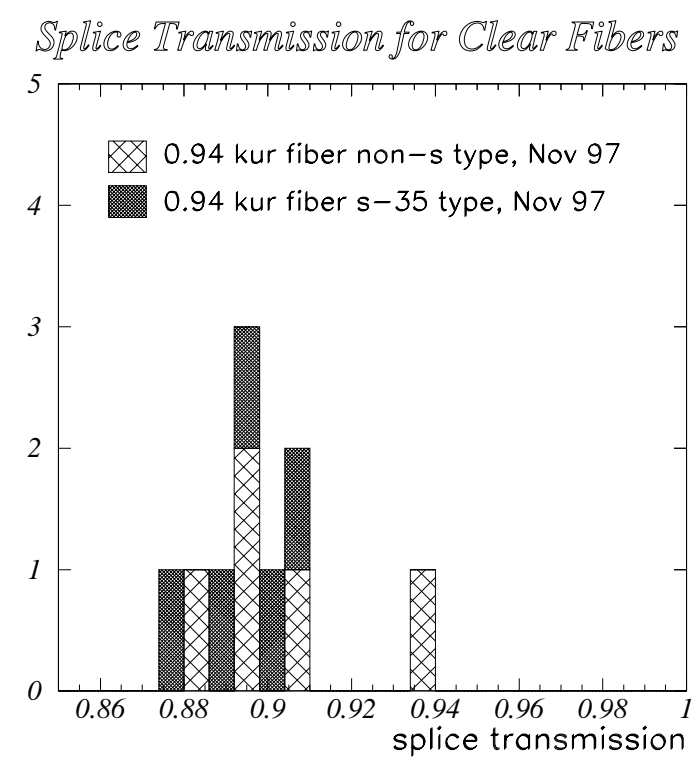

FIGURE 5. Mirror reflectivity measured with the UV scanner. (a) shows the measurement for Kuraray fibers. "Avtech" means the fibers are polished with the Avtech polisher, and "Ice" means the fibers are ice polished. (b) compares the reflectivity between Kuraray, $0.83 \mathrm{~mm}$, non-S fiber and Bicron 0.83 multiclad fiber. The fibers for (b) used the Avtech polisher.
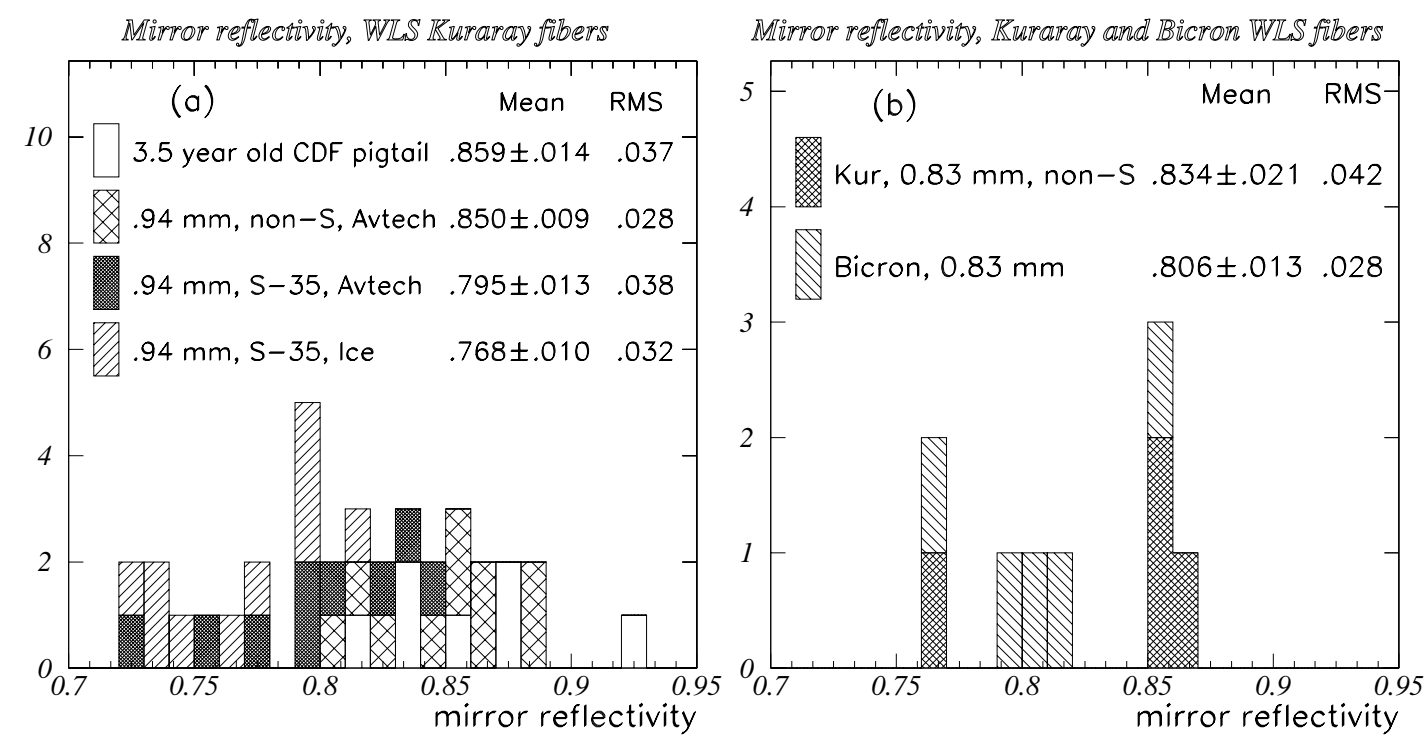
FIGURE 6. Setup to determine the light increase from a mirror in a tile.

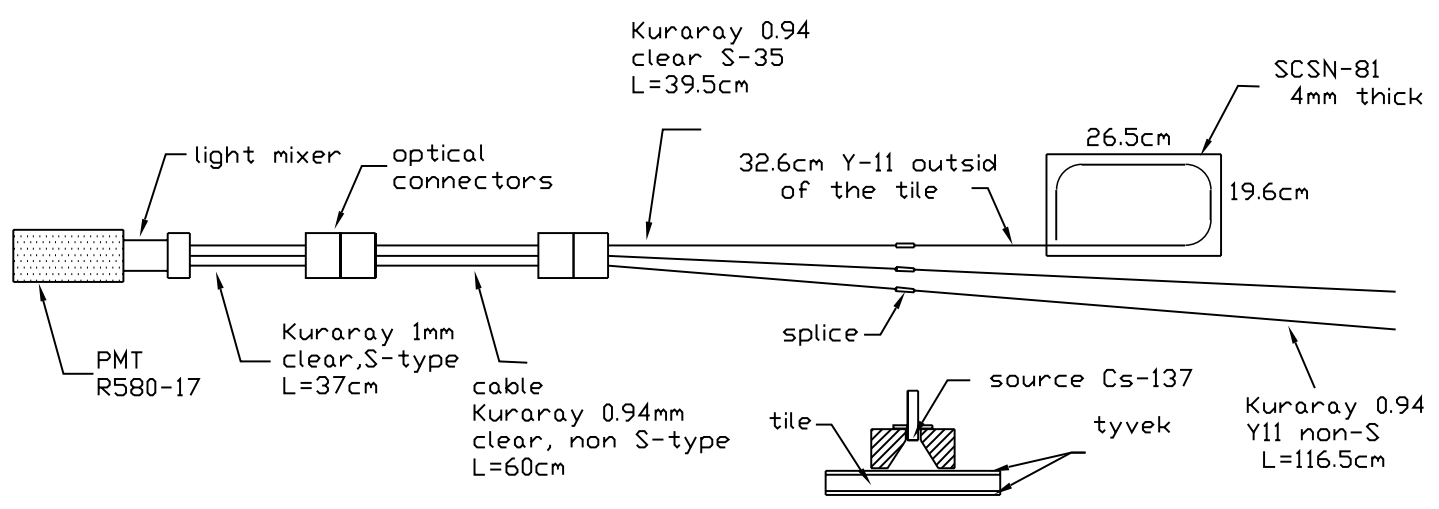

reflectivity measurements done using a scintillator tile. Figure 5a states that light increase from the mirror for ice polished S-35 fibers from the UV scanner is 1.77 . The corresponding increase for a tile is 1.53 .

\section{RELATIVE LIGHT YIELD FOR DIFFERENT FIBERS}

We measured the light response of different kinds of fibers. Figure 7 shows the setup used for the relative light yield test. Fibers are inserted into a tile made of Kuraray SCSN-81 scintillator. A clear "cable" connects the pigtail from the connector to the phototube. Table 3 gives the result for different types of WLS fibers using both $103 \mathrm{~cm}$ and $251 \mathrm{~cm}$ Kuraray $1 \mathrm{~mm}$ S type clear cables. Column 2 and 3 are separately normalized to the Kuraray 0.83 $\mathrm{mm}, 250 \mathrm{ppm}$, non-S result (labeled by $\equiv$ ). For Kuraray fiber, the $0.94 \mathrm{~mm}$ WLS fiber yields $6 \%$ more light than a $0.83 \mathrm{~mm}$ WLS fiber. There is no difference in light between a $0.94 \mathrm{~mm}$ WLS fiber and a $1.0 \mathrm{~mm}$ WLS fiber connected to $1 \mathrm{~mm}$ clear fiber. Table 4 gives the core diameter and fiber diameter. One sample of each fiber is measured. The increase in light is smaller than the increase in either the core diameter or the fiber diameter.

\section{ATTENUATION LENGTH OF CLEAR FIBERS}

We have looked at the relative light transmission of different clear fibers by using two different cables. Figure 7 shows the setup. Table 5 gives the ratio of light for the two different cables. Column 2 and column 3 give the cables used. The length of the cables for column 2 are all $251 \mathrm{~cm}$. Column 
FIGURE 7. Setup for relative light yield test. One piece of each hardware is made except for the $251 \mathrm{~cm}$ cables Kuraray $1 \mathrm{~mm}$ S type where 2 are made. Results are shown in Table 3 and 5.

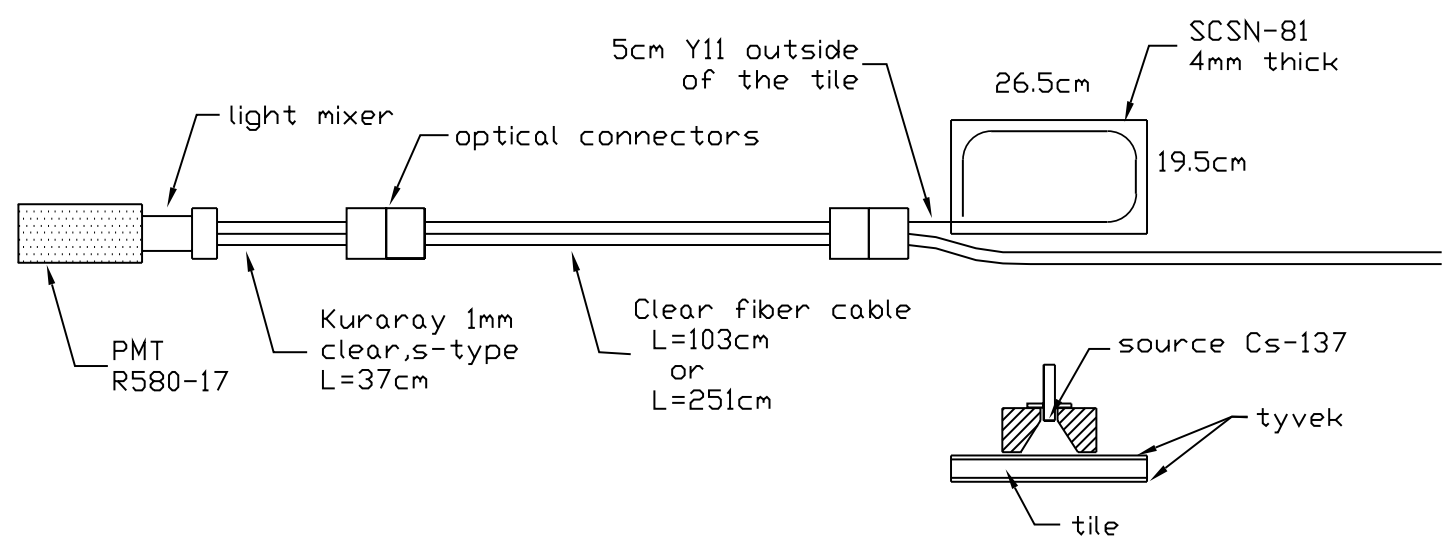

TABLE 3. Relative light yield. The cables are made of $1 \mathrm{~mm} \mathrm{~S}$ type Kuraray fiber. Column 2 gives the light using a $251 \mathrm{~cm}$ cable and Column 3 gives the light using a $103 \mathrm{~cm}$ cable. The entries marked $\equiv$ are defined to be 1.00 .

\begin{tabular}{|l|c|c|}
\hline Type of WLS fiber & 251 cm Cable & $103 \mathrm{~cm}$ Cable \\
\hline Bicron 1.00 mm, 200 ppm, batch 1 & 0.93 & 0.93 \\
Bicron 1.00 mm, 200 ppm, batch 2 & 1.00 & 1.02 \\
Kuraray 0.83 mm, 250 ppm, non-S & $\equiv 1.00$ & $\equiv 1.00$ \\
Kuraray 0.94 mm, 250 ppm, S-35 & 1.07 & 1.06 \\
Kuraray 0.94 mm, 250 ppm, S-50 & 1.06 & 1.06 \\
Kuraray $1.00 \mathrm{~mm}, 200 \mathrm{ppm}$, non-S & 1.06 & 1.05 \\
Kuraray $1.00 \mathrm{~mm}, 300 \mathrm{ppm}, \mathrm{S}$ & 1.08 & 1.07 \\
\hline
\end{tabular}

TABLE 4. Core and fiber diameter. All units are mm. One fiber is measured for each type. $\dagger$ means fiber has only one visible cladding. $\ddagger$ means interface between outer cladding and inner cladding was not distinguishable enough to measure it. Kuraray fiber has 2 claddings. Multiclad Bicron fiber has one visible cladding, as the outer cladding is too thin to be visible.

\begin{tabular}{|l|c|c|c|}
\hline Fiber type & Core diameter & Inner cladding diameter & Outside diameter \\
\hline Bicron, 0.83 mm, WLS & 0.789 & $\dagger$ & 0.850 \\
Kuraray, 0.83 mm non-S, WLS & 0.742 & 0.786 & 0.844 \\
Kuraray, 0.83 mm non-S, clear & 0.737 & $\ddagger$ & 0.838 \\
Kuraray, 0.94 mm non-S, WLS & 0.841 & 0.903 & 0.959 \\
Kuraray, 0.94 mm non-S, clear & 0.838 & $\ddagger$ & 0.946 \\
Kuraray, 1.00 mm non-S, WLS & 0.887 & 0.955 & 1.008 \\
Kuraray, 1.00 mm non-S, clear & 0.902 & 0.958 & 1.010 \\
\hline
\end{tabular}


TABLE 5. Comparison of light yields using two different clear cables.

\begin{tabular}{|l|l|l|c|}
\hline Pigtail & Cable 1, L=251 cm & Cable 2 & Cab 1/Cab 2 \\
\hline Kur 1.00 mm, non-S & Kur 1.00 mm S & $103 \mathrm{~cm} \mathrm{Kur} \mathrm{1.00} \mathrm{mm} \mathrm{S}$ & 0.79 \\
Kur 0.94 mm, S-35 & Kur 0.94 mm S-35 & $103 \mathrm{~cm}$ Kur 0.94 mm S-35 & 0.80 \\
Kur 0.94 mm, S-50 & Kur 0.94 mm S-50 & $103 \mathrm{~cm}$ Kur 0.94 mm S-50 & 0.80 \\
Bicron 1.00 mm & Bicron 1.00 mm & $251 \mathrm{~cm}$ Kur 1.00 mm S & 0.89 \\
\hline
\end{tabular}

FIGURE 8. Setup to measure attenuation length of cables of Kuraray, $0.9 \mathrm{~mm}$, S-type clear fibers. Results given in Figure 10a. Pigtails A and B measure different clear fibers.

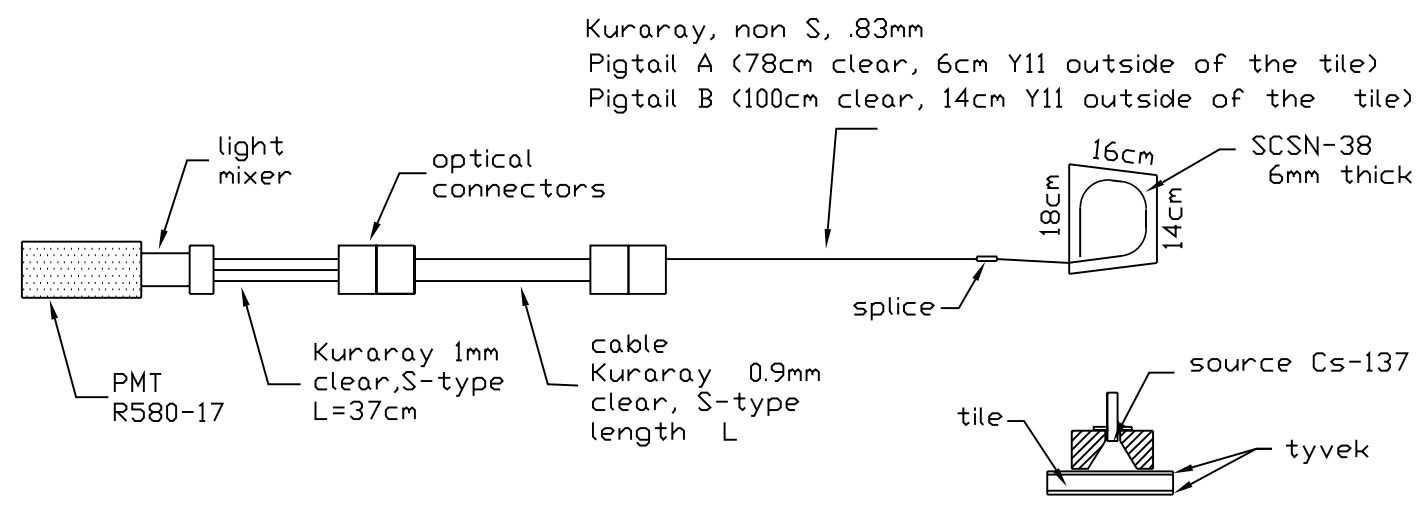

4 gives the ratio of the measurement of column 2 over column 3. The results are consistent with an equal attenuation length for $1 \mathrm{~mm} \mathrm{S,} 0.94 \mathrm{~mm} \mathrm{S-35,}$ and 0.94 S-50 Kuraray fiber.

We have measured the attenuation length of the clear fiber in a cable. Figure 8 gives the apparatus for measuring Kuraray $0.9 \mathrm{~mm}$ S type fibers, and Figure 10a gives the result. Each point is a measurement of one fiber in a cable. Two separate pigtails are used and they give the same attenuation length of the clear fiber. The combined results of both pigtails for the attenuation length is $732 \pm 13 \mathrm{~cm}$. The RMS of the normalized light about the exponential curve in Figure 10a is 5.6\%. The test shown in Figure 9 measures the difference in attenuation between the Kuraray $1 \mathrm{~mm}$ fiber and Bicron $1 \mathrm{~mm}$ fiber. Figure $10 \mathrm{~b}$ gives the result. Each point is the average of the three fibers in the pigtail. The attenuation lengths for Kuraray fibers given in Figure 10a and Figure 10b agree.

The data for the clear fiber attenuation measurements are fit to $a e^{-x / b}$. The data and curve are normalized by setting $\mathrm{a}=1$. A fit to the data used for Figure $10 \mathrm{~b}$ gives $\mathrm{a}_{k u r} / \mathrm{a}_{b i c}=1.02 . \mathrm{a}_{k u r} / \mathrm{a}_{b i c}$ is the amount of light accepted by the Kuraray clear fibers divided by the amount of light accepted by the 
FIGURE 9. Apparatus to measure the attenuation length of $1 \mathrm{~mm}$ clear fiber cables. Bicron multiclad and Kuraray S-type fibers are measured. The same cables are measured with both pigtails. Results given in Figure 10b.

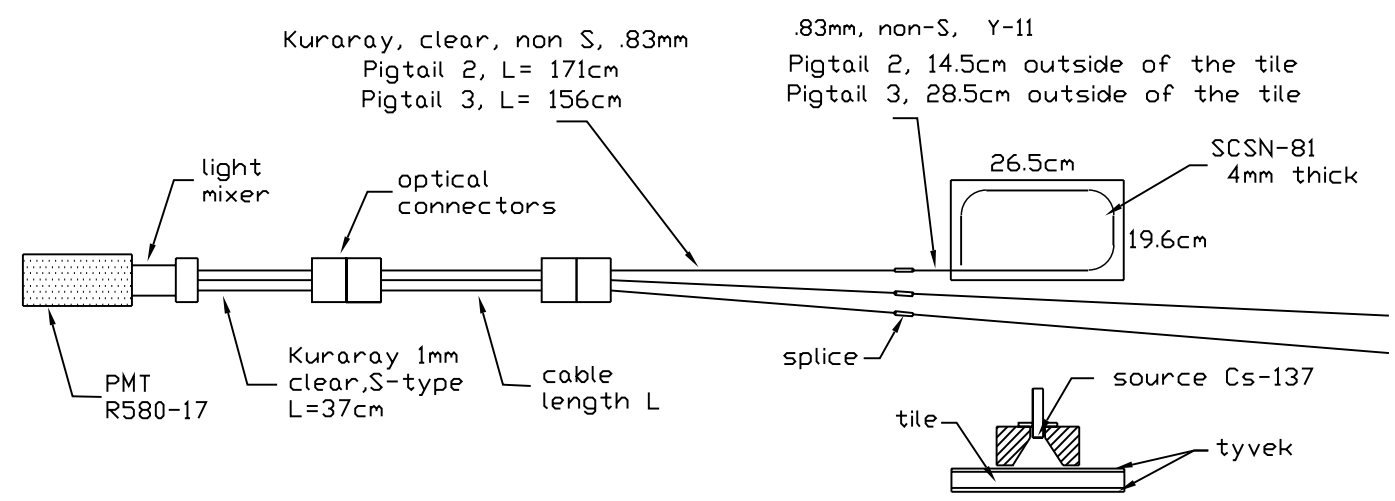

FIGURE 10. Attenuation length of clear fibers.
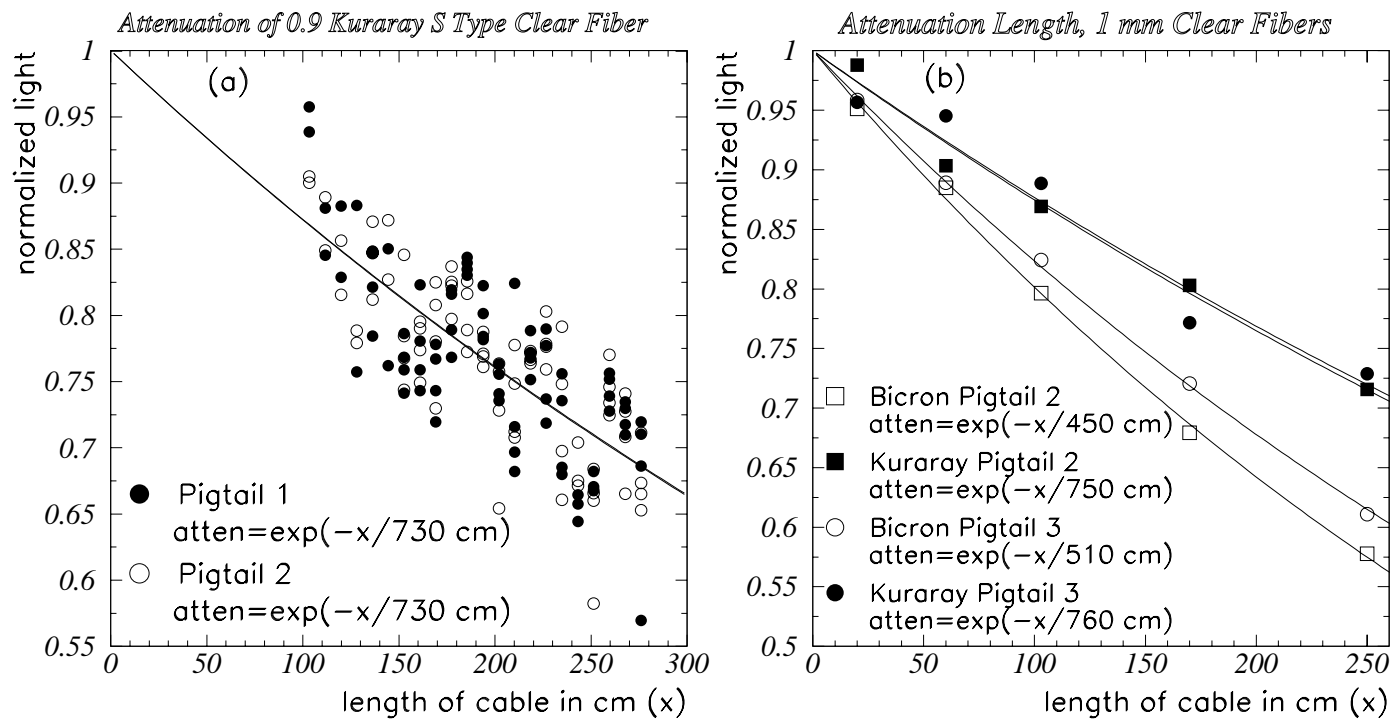

Bicron clear fibers. Theoretically, this should be the same as ratio of numerical apertures of the two kinds of fibers. Hence, Kuraray fiber and Bicron fiber have the same numerical aperture.

We have looked at the attenuation length of the clear fiber when one end is spliced to a WLS fiber and the other end is glued in a connector. Figure 11 shows the apparatus used to measure the clear attenuation length, and Figure 12 gives the results. For Test A ( Figure 12a), one pigtail with 3 fibers is made for each length of clear fiber. A single exponential fit gives $\lambda=6.4 \mathrm{~m}$ with the cable and $\lambda=6.55 \mathrm{~m}$ without the cable. Hence, the attenuation length of 
FIGURE 11. Apparatus used to measurement of attenuation length of clear fiber spliced to WLS fibers. Results given in Figure 12.

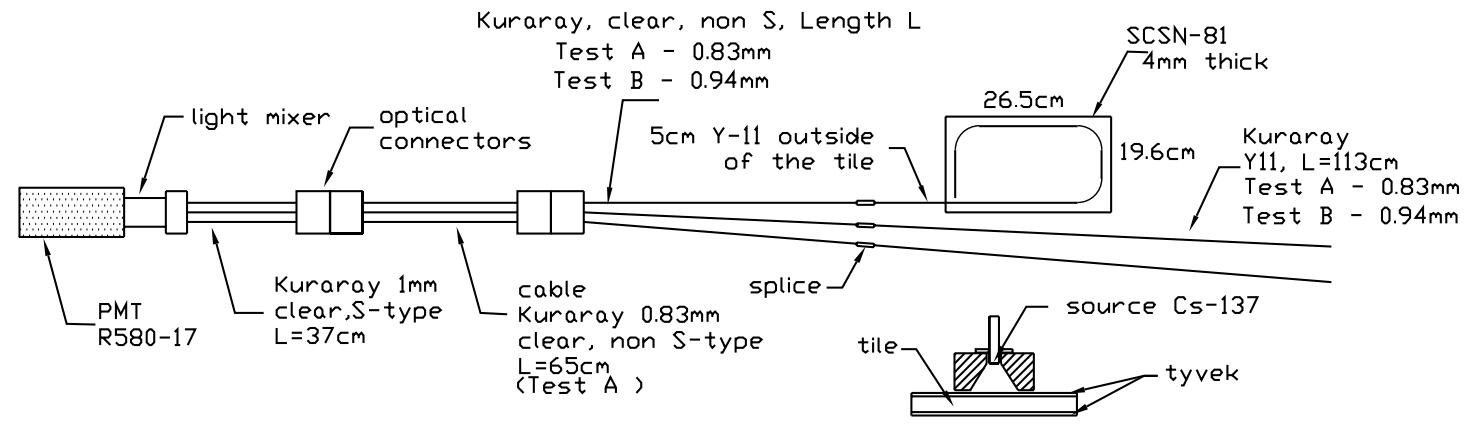

FIGURE 12. Attenuation length of clear fiber spliced to a WLS fiber in a pigtail.
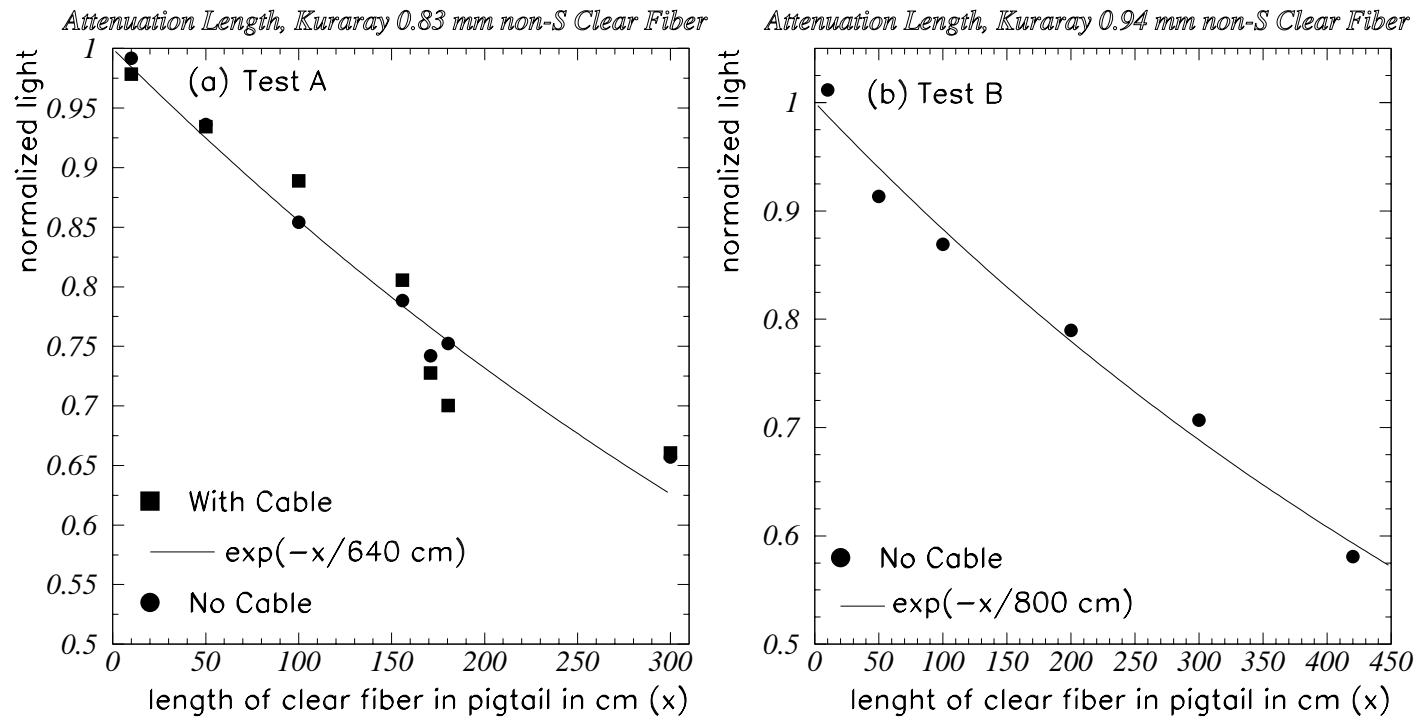

the clear fiber does not depend on whether the cable is present.

For Test B (Figure 12b), one pigtail was made with three $4.2 \mathrm{~m}$ clear fibers. The pigtail is measured. The pigtail connector is cut off and a new connector is put on with the clear fiber reduced to $3 \mathrm{~m}$. The pigtail is measured and the process continues until the pigtail has $0.1 \mathrm{~m}$ of clear fiber left. Test B uses the same splice between the clear and green fibers for all the clear lengths and should give a better measurement of the attenuation length.

The measurement shown in Figure 10a gives the best measurement of the attenuation length of the clear Kuraray fiber. A single exponential with $\lambda=$ $7.3 \mathrm{~m}$ gives an adequate description of the attenuation length of all Kuraray 
FIGURE 13. Attenuation length of different WLS fibers. The results measured with the MINOS setup are shown in (a), and the results measured with the UV scanner shown in (b). The numbers after the symbols in the plots state the number of fibers measured.
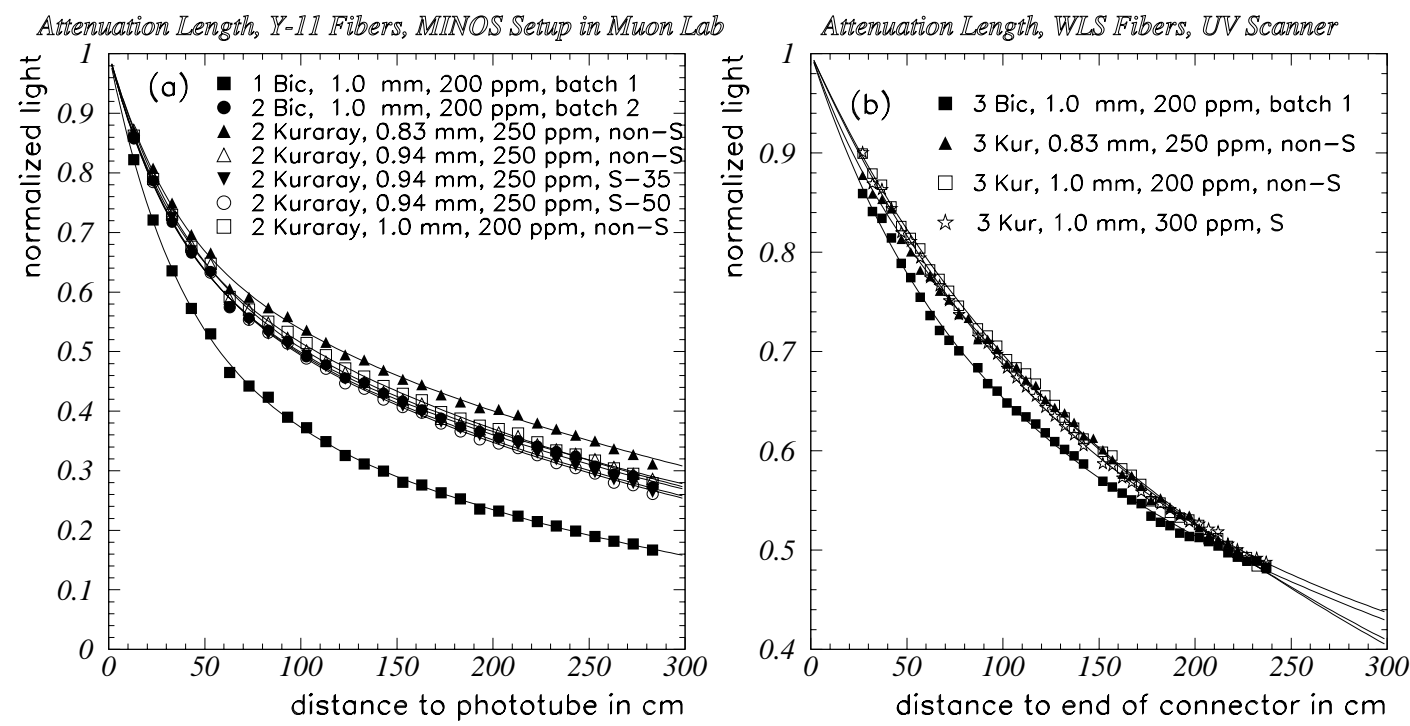

FIGURE 14. A schematic of the MINOS setup to measure fiber attenuation.

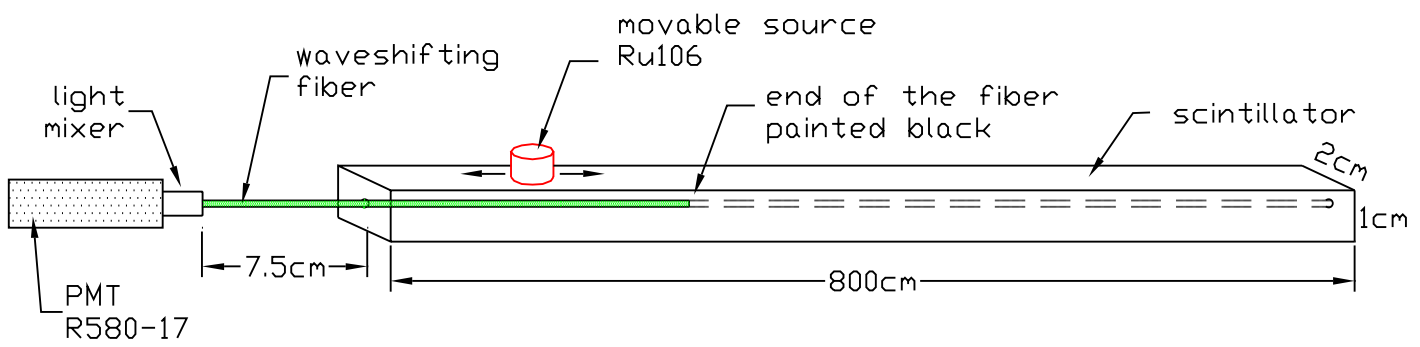

clear fiber for lengths $<4 \mathrm{~m}$. The other measurements of the clear Kuraray fiber are consistent with this measurement.

\section{ATTENUATION LENGTH OF WLS FIBERS}

We have measured the attenuation lengths of various WLS fibers. The attenuation lengths are measured with a setup provided by the MINOS experiment, see Figure 14 . The MINOS setup provides an easy and quick way of measuring the attenuation length of many WLS fibers using scintillator material as a light source. One end of the fiber is polished, and the other end is cut at $45^{\circ}$ and painted black. The fiber is inserted into a long hole in 
FIGURE 15. Comparison of the attenuation length of Kuraray $0.94 \mathrm{~mm}, 250 \mathrm{ppm}$, non-S measured with $3 \mathrm{~m}$ and $4 \mathrm{~m}$ pieces.

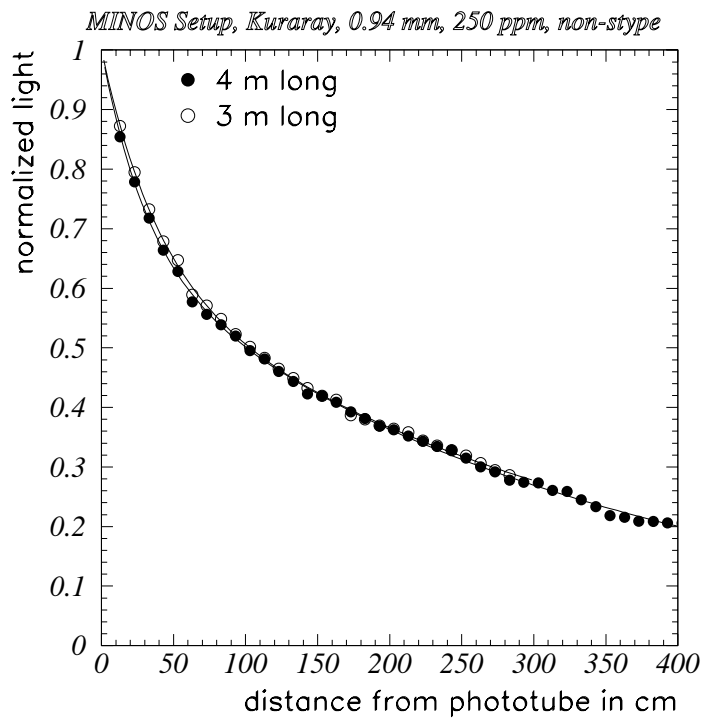

the scintillator and the polished end is pushed up against a light mixer on the phototube. The source is moved across the scintillator and the phototube current is read out with a picoammeter. The data are fit to $a e^{-x / l_{1}}+b e^{-x / l_{2}}$. Figure 13a plots the distance from source to the phototube vs the normalized light. Table 6 gives the numerical results of the fits.

Almost all of our WLS fibers are $3 \mathrm{~m}$ long. We have some $4 \mathrm{~m}$ Kuraray $0.94 \mathrm{~mm}, 250 \mathrm{ppm}$, non-S fiber. To determine if the attenuation lengths using $3 \mathrm{~m}$ and $4 \mathrm{~m}$ pieces agree, we measure two $4 \mathrm{~m}$ pieces in the MINOS setup. We cut the 2 pieces to $3 \mathrm{~m}$ and remeasured them. Figure 15 plots the results. The measurement shows that an attenuation length measured with a 3 meter piece can be extrapolated to $4 \mathrm{~m}$.

We compared the above measurement with a measurement using a UV light and pin diodes to read out the fibers, see Figure 1. Figure 13b plots the attenuation of the fibers vs the distance to the connector. The MINOS measurement gives a greater difference between the Kuraray fiber and batch 1 Bicron fiber than the UV measurement. The greater sensitivity of pin diodes in the UV setup to long wavelengths light may be the reason. Table 6 gives the results of the fits in the rows labeled UV.

Fibers can be measured quickly with either the MINOS setup or UV scanner. Both tests are useful for comparing fibers. However, the setups may not give the correct attenuation length of the WLS fibers which are relevant for CMS HCAL design. Figure 16 shows the setup designed to give a more accurate attenuation length. Figure 17a shows the attenuation length of two kinds of fibers measured with the sliding fiber setup. We have shown the measurements 
TABLE 6. Results of fit to attenuation data for WLS fibers. The data are fit to $a e^{-x / l_{1}}+b e^{-x / l_{2}}$. All units for $l_{1}$ and $l_{2}$ are $\mathrm{cm}$. The column marked Setup gives the apparatus used to measure the fibers. MINOS uses the MINOS apparatus, UV used the CDF UV scanner, and SLIDE is the sliding fiber apparatus. MINOS RAD are the radiated fibers measured with the MINOS setup. BEFORE RAD are the same fibers measured before irradiation with the MINOS apparatus with the correction function. UV RAD are the same radiated fibers measured with the UV setup. These are the same fibers labeled UV in the Setup column.

\begin{tabular}{|l|c|c|c|c|c|}
\hline WLS Fiber type & Setup & $a$ & $l_{1}$ & $b$ & $l_{2}$ \\
\hline Bicron 1.00 mm, 200 ppm, bat-1 & MINOS & 0.488 & 35 & 0.512 & 254 \\
Bicron 1.00 mm, 200 ppm, bat-2 & MINOS & 0.357 & 33 & 0.643 & 343 \\
Kuraray 0.83 mm, 250 ppm, non-S & MINOS & 0.323 & 34 & 0.677 & 379 \\
Kuraray 0.94 mm, 250 ppm, non-S & MINOS & 0.372 & 39 & 0.628 & 366 \\
Kuraray 0.94 mm, 250 ppm, S-35 & MINOS & 0.345 & 33 & 0.655 & 320 \\
Kuraray 0.94 mm, 250 ppm, S-50 & MINOS & 0.348 & 34 & 0.652 & 317 \\
Kuraray 1.00 mm, 200 ppm, non-S & MINOS & 0.317 & 31 & 0.683 & 326 \\
Bicron 1.00 mm, 200 ppm, bat-1 & UV & 0.304 & 63 & 0.696 & 611 \\
Kuraray 0.83 mm, 250 ppm, non-S & UV & 0.102 & 33 & 0.898 & 375 \\
Kuraray $1.00 \mathrm{~mm}, 200 \mathrm{ppm}$, non-S & UV & 0.188 & 79 & 0.812 & 431 \\
Kuraray $1.00 \mathrm{~mm}, 300 \mathrm{ppm}, \mathrm{S}$ & UV & 0.524 & 131 & 0.476 & 1407 \\
Kuraray $0.94 \mathrm{~mm}, 250 \mathrm{ppm}, \mathrm{S}-35$ & SLIDE & 0.287 & 31 & 0.713 & 366 \\
Bicron $1.00 \mathrm{~mm}, 200 \mathrm{ppm}$, bat-1 & SLIDE & 0.381 & 31 & 0.619 & 303 \\
Bicron $1.00 \mathrm{~mm}, 200 \mathrm{ppm}$, bat-1 & MINOS RAD & 0.484 & 30 & 0.516 & 259 \\
Bicron $1.00 \mathrm{~mm}, 200 \mathrm{ppm}$, bat-1 & BEFORE RAD & 0.462 & 39 & 0.538 & 332 \\
Kuraray $1.00 \mathrm{~mm}, 200 \mathrm{ppm}$, non-S & MINOS RAD & 0.343 & 33 & 0.657 & 250 \\
Kuraray $1.00 \mathrm{~mm}, 200 \mathrm{ppm}$, non-S & BEFORE RAD & 0.335 & 42 & 0.665 & 361 \\
Kuraray $1.00 \mathrm{~mm}, 300 \mathrm{ppm}, \mathrm{S}$ & MINOS RAD & 0.362 & 31 & 0.638 & 235 \\
Kuraray $1.00 \mathrm{~mm}, 300 \mathrm{ppm}, \mathrm{S}$ & BEFORE RAD & 0.331 & 36 & 0.669 & 323 \\
Bicron $1.00 \mathrm{~mm}, 200 \mathrm{ppm}$, bat-1 & UV RAD & 0.305 & 58 & 0.695 & 358 \\
Kuraray $1.00 \mathrm{~mm}, 200 \mathrm{ppm}$, non-S & UV RAD & 0.395 & 148 & 0.605 & 332 \\
Kuraray $1.00 \mathrm{~mm}, 300 \mathrm{ppm}, \mathrm{S}$ & UV RAD & 0.733 & 152 & 0.267 & 1056 \\
\hline
\end{tabular}

of the same fibers with the MINOS measurement. Table 6 gives the results for the sliding fiber test for those entries marked SLIDE in column 2. The results of the sliding fiber setup for $0.94 \mathrm{~mm}$ Kuraray are used in the design of CMS HCAL.

Figure 17a shows the difference in attenuation length of a fiber measured in two different ways, but with the same photodetector and light injection. The difference seen is due to the extra clear fiber cable and the clear to WLS splice of the sliding fiber setup. Figure 17b compares the sliding tile measurement with the MINOS measurement with $\mathrm{x}=0$ set $18 \mathrm{~cm}$ (Kuraray) and $25 \mathrm{~cm}$ (Bicron) away from the phototube into the scintillator. The comparison shows $18 \mathrm{~cm}$ (Kuraray) of WLS fiber is acting like the clear fiber and cable. 
FIGURE 16. Sliding fiber setup. The results are shown in Figure 17.

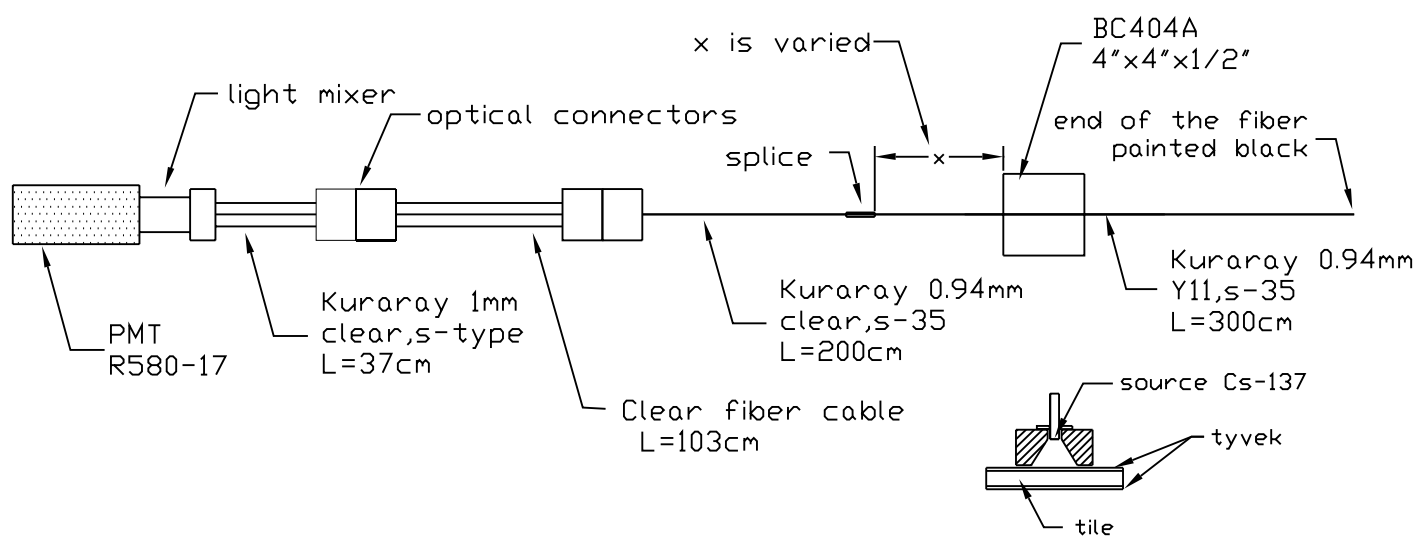

FIGURE 17. Attenuation measured using sliding fiber setup and compared with the MINOS measurement. (b) put the $\mathrm{x}$ origin for the MINOS measurement $18 \mathrm{~cm}$ (Kuraray) and $25 \mathrm{~cm}$ (Bicron) into the fiber.
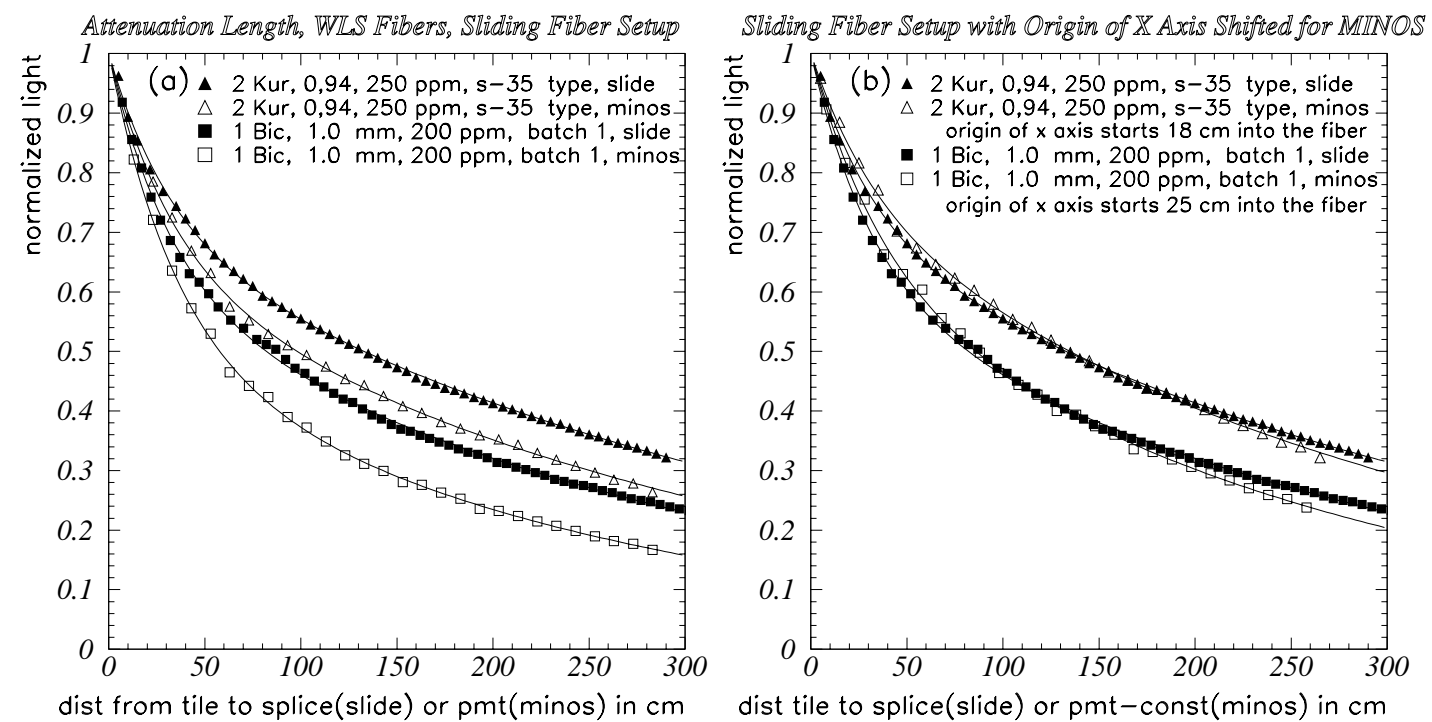

\section{RADIATION STUDY OF FIBERS}

Some of the WLS fibers were irradiated with $127 \mathrm{krad}$ at an electron source at Florida State University.

We encountered a problem in measuring the irradiated fibers. The fibers were first measured with the MINOS setup in Lab 5 at Fermilab. While the fibers were being irradiated, the MINOS setup was moved to the Muon Lab. We measured the irradiated fibers with the MINOS setup in Muon Lab. 
FIGURE 18. Comparison of attenuation length of WLS fibers before and after irradiation with $127 \mathrm{Krad}$. The fiber diameters are $1.0 \mathrm{~mm}$. The 2 setups to measure the fibers are (a) MINOS setup and (b) CDF UV Scanner.
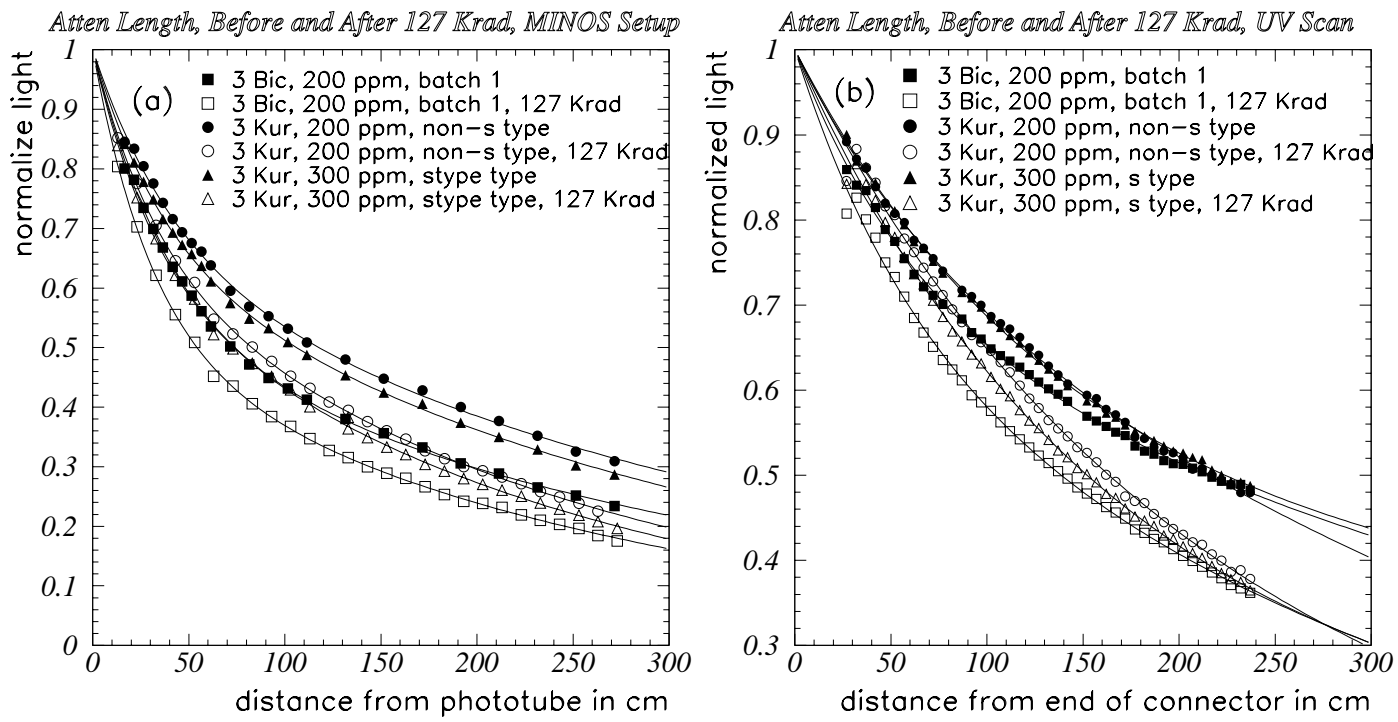

We measured the same three Kuraray $0.83 \mathrm{~mm}$ fibers in both Lab 5 and the Muon Lab. The normalized light at $100 \mathrm{~cm}$ was .57 in Lab 5 and .52 in the Muon Lab. We have no explanation for the difference, since the setups are the same. The normalized light difference between different fiber types is not affected by this problem, but the absolute normalized light is affected by this problem. The measurement of the $0.83 \mathrm{~mm}$ fiber in both Lab 5 and Muon Lab is used to get a correction function for the Lab 5 measurement. The normalized light yield of the fibers before irradiation is multiplied by the correction function. Figure 18 compares the normalized light of the fibers before and after radiation measured with both the MINOS setup and the CDF UV scanner. The results of the fits of the fibers before and after radiation are given in Table 6.

\section{LIGHT VS TILE SIZE AND ABSOLUTE LIGHT}

Figure 19 and Figure 20 show the setup used to measure the light vs tile size for CMS HCAL tower 10 and 16, respectively. Tower 10 is a tower in the middle of the barrel, while tower 16 is at the high eta edge of the barrel. To measure tower 10, we make 6 tiles each for layers 1, 7, and 16, for a total of 18 tiles. We use the same pigtail to measure the light from each of the tiles. The results are plotted as light vs perimeter/area [11] [12]. The perimeter measures the length of fiber in the tile, since the distance the fiber groove is 
FIGURE 19. Setup used to measure relative light vs tile size for Tower 10. The results are given in Figure 21.

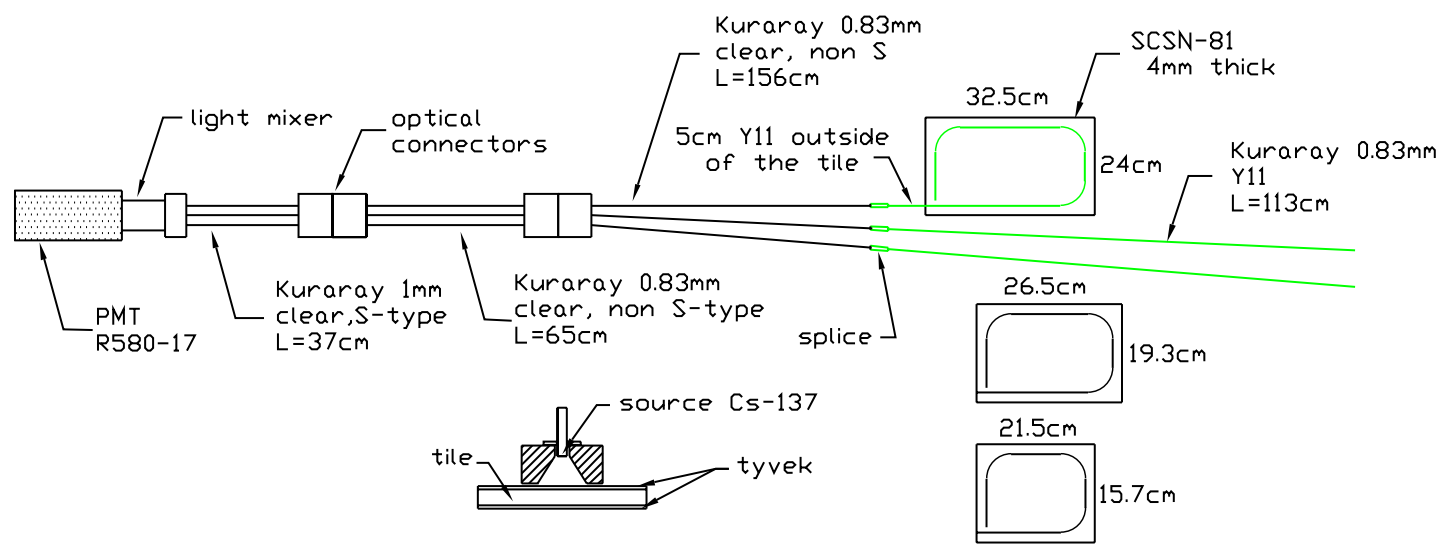

from the edge of the scintillator is kept at $0.3 \mathrm{~cm}$ for these measurements. Figure 21a gives the result. This result includes the additional length of WLS fiber outside the tile for layers 1 and 7 . Figure 21b gives the result with the WLS fiber attenuation removed. Figure $21 \mathrm{~b}$ gives the light vs tile size. The overall normalization is not measured by this measurement. The mean of the data for tower 10 is normalized to 1 for both Figure 21a and Figure 21b. The data on Figure $21 \mathrm{~b}$ is fit to a straight line. The 3.8 is a relative number that depends on the how the light measurement is normalized. The measurement measures the coefficent $0.077 / \mathrm{cm}$, which is the intercept divided by the slope of the line.

To measure tower 16, we make 5 tiles each for layers 1, 5, and 8. Again, we use the same pigtail to measure the light from each of the tiles. The pigtails for the tower 10 measurement and tower 16 measurement are different. Hence, the normalization of the tower 10 and tower 16 measurements are independent. The mean of the data for tower 16 in Figure 21a is set to 1. For Figure 21b the normalization for tower 16 is set so that the mean of the perimeter/area and mean of the normalized light lie on the straight line for tower 10. This enables us to see how consistent the two measurements are.

For the CMS design we used two models for the variation of light vs tile size. The first model assumes the light yield is a linear function of perimeter/area. The line is given in Figure 21b. We notice that the points for Tower 16 do not follow a straight line. In the measurement for Figure 21a the same fibers are used to measure all the tiles in a tower. The total variation of the points for Figure 21a for both Tower 10 and 16 is $4 \%$. The second model assumes that a fiber with the same length green inserted into all the CMS tiles gives the same light. It assumes the change in the light by changing the tile size is compensated for by the attenuation in the green fiber.

Figure 2222 shows the apparatus used to measure the absolute light yield of CMS tiles. A Ru-106 $\beta$ source is used instead of the Cs-137 source. Figure 23 
FIGURE 20. Setup used to measure relative light vs tile size for Tower 16 . The results are given in Figure 21.

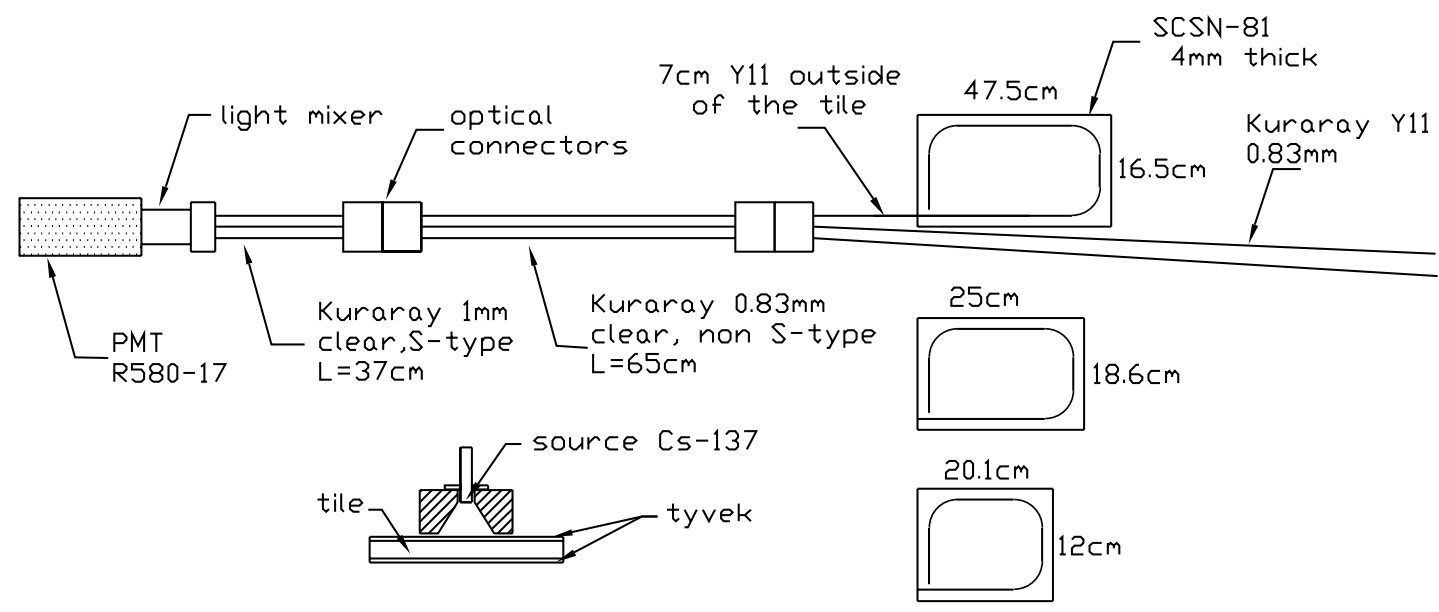

FIGURE 21. Relative light vs tile size. (b) has the attenuation from the WLS fiber between the tile and splice removed.
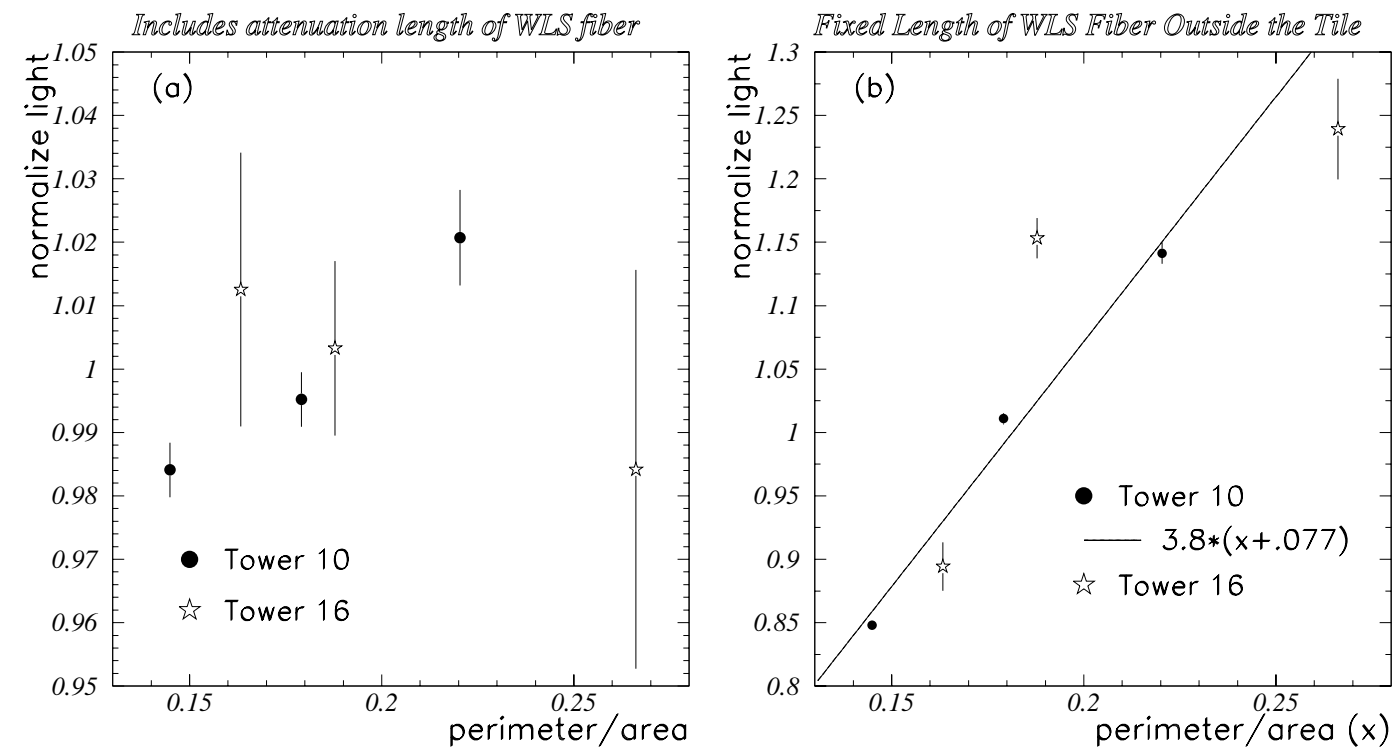
FIGURE 22. Setup to measure absolute light from a tile in CMS. Results are given in

Figure 23.

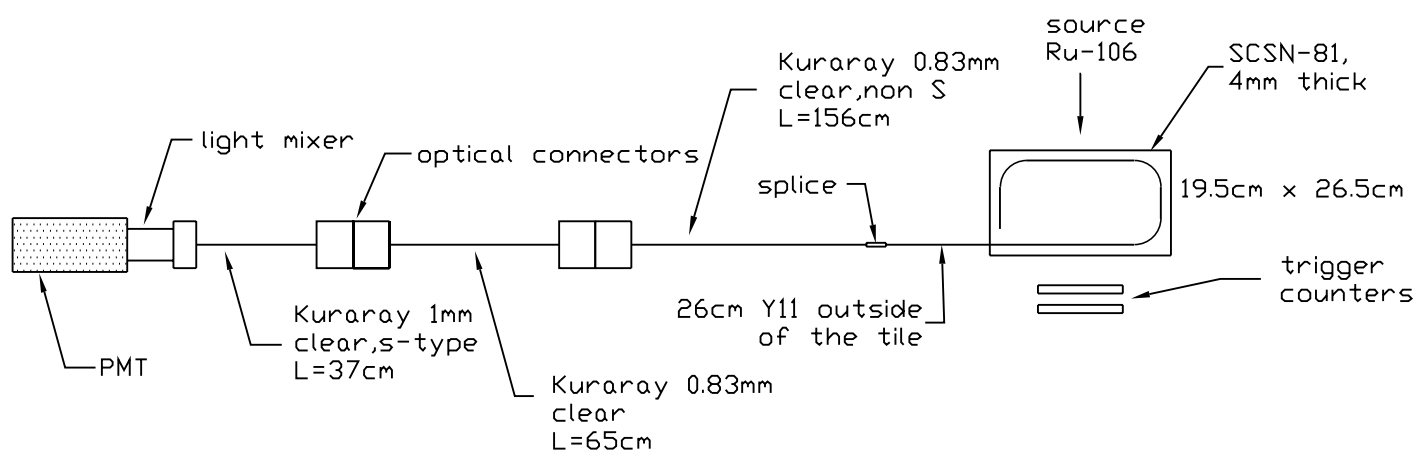

FIGURE 23. Absolute light yield.
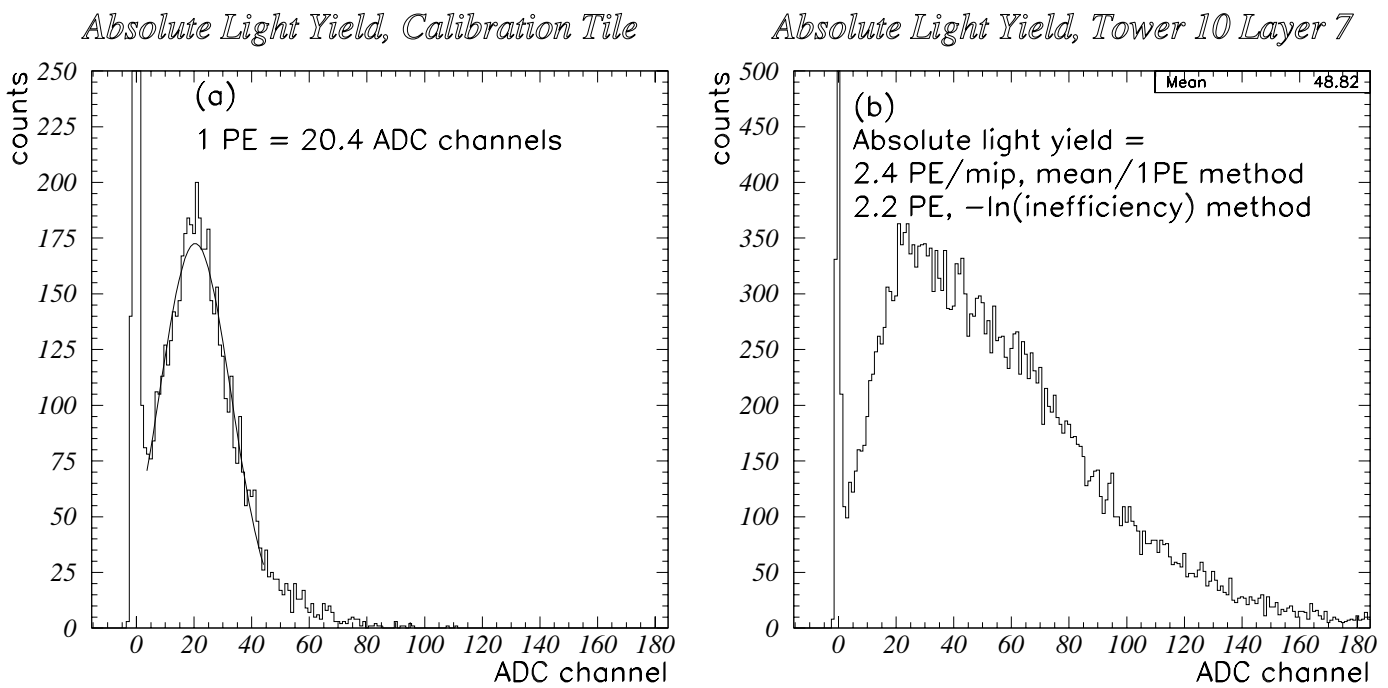

gives the result. A CMS HCAL barrel tile gives roughly 2 photoelectrons at the photodetector with a green extended photocathode.

The light for the tiles in HCAL barrel can be predicted using the attenuation length of the green and clear fiber, the model of the light vs tower size, and the absolute light yield. From this we can get the total light of a tower. All layers of a tower, except for the first, go to the same photodetector. The longitudinal variation of light within a tower should be less than 10\%. By varying the position of the splice, we make the light uniform longitudinally in a tower. 
FIGURE 24. Apparatus to measure transverse tile uniformity with $1 \mathrm{~mm}$ air gap between the tiles. The fibers are inserted parallel to the short side. The result is shown in Figure 26.

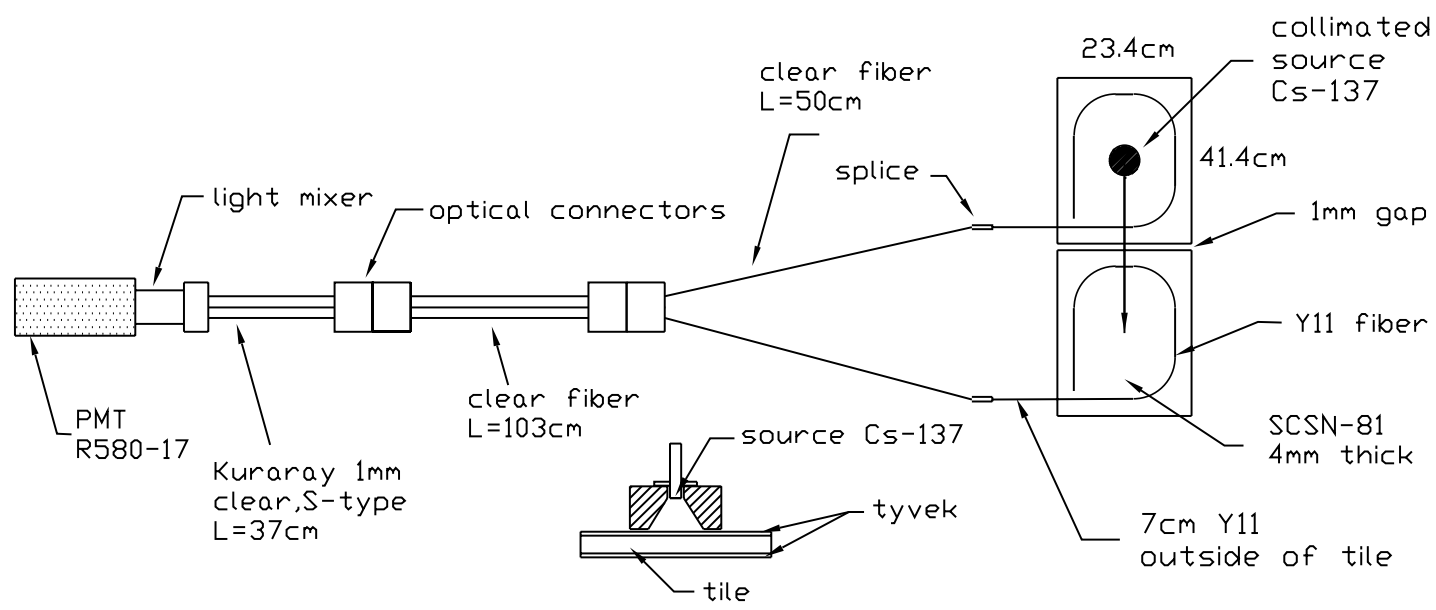

\section{TRANSVERSE TILE UNIFORMITY}

We have studied the transverse uniformity of the tiles. We constructed four tiles with the dimensions of tower 14, layer 14, which is the largest tile in the CMS HCAL Barrel. Two tiles have the fibers inserted parallel to the short side, called short side fiber insertion. The tiles with short side fiber insertion are shown in Figure 24. Two tiles have the fibers inserted parallel to the long side, called long side fiber insertion. The tiles for long side fiber insertion are shown in Figure 25. CMS HCAL tiles have long side fiber insertion. The edges of the tiles are painted with white $\mathrm{TiO}_{2}$ paint [13].

The uniformity is measured with a collimated Cs-137 $\gamma$ source. The collimator constrains the radiation to a $7.5 \mathrm{~cm}$ diameter circle. The central transverse size of a hadron shower is approximately $7.5 \mathrm{~cm}$. Hence, the collimated source simulates the transverse size of a hadron shower. Figure 26 shows the uniformity across the tile with the short side fiber insertion. The uniformity was measured with both Kuraray fibers and Bicron fibers. The Kuraray measurement uses $0.94 \mathrm{~mm} \mathrm{S-35}$ fibers for both the pigtail and the cable. For the Bicron measurement, the WLS 1.0 mm Bicron fiber (Batch 2) is spliced to 1.0 $\mathrm{mm}$ non-S Kuraray fiber. The cable for the Bicron measurement was made with $\mathrm{S}$ type $1.0 \mathrm{~mm}$ Kuraray fiber. For both kinds of fibers the tile is very uniform with a $10 \%$ increase at the boundary between the 2 tiles. The increase is due to increased light collection at the fiber. Figure 27 shows the uniformity with long side fiber insertion. The transverse uniformity has an RMS $\sim 3 \%$ regardless of the fiber type or fiber insertion point. The resolution of the CMS calorimeter is $120 \% / \sqrt{E} \oplus 5 \%$ [14]. The transverse uniformity across the tile should be somewhat less than the constant term, $5 \%$, to prevent transverse 
FIGURE 25. Apparatus to measure transverse tile uniformity with $1 \mathrm{~mm}$ air gap between the tiles. The fibers are inserted into the tile parallel to the scan. The measurement is shown in Figure 27.

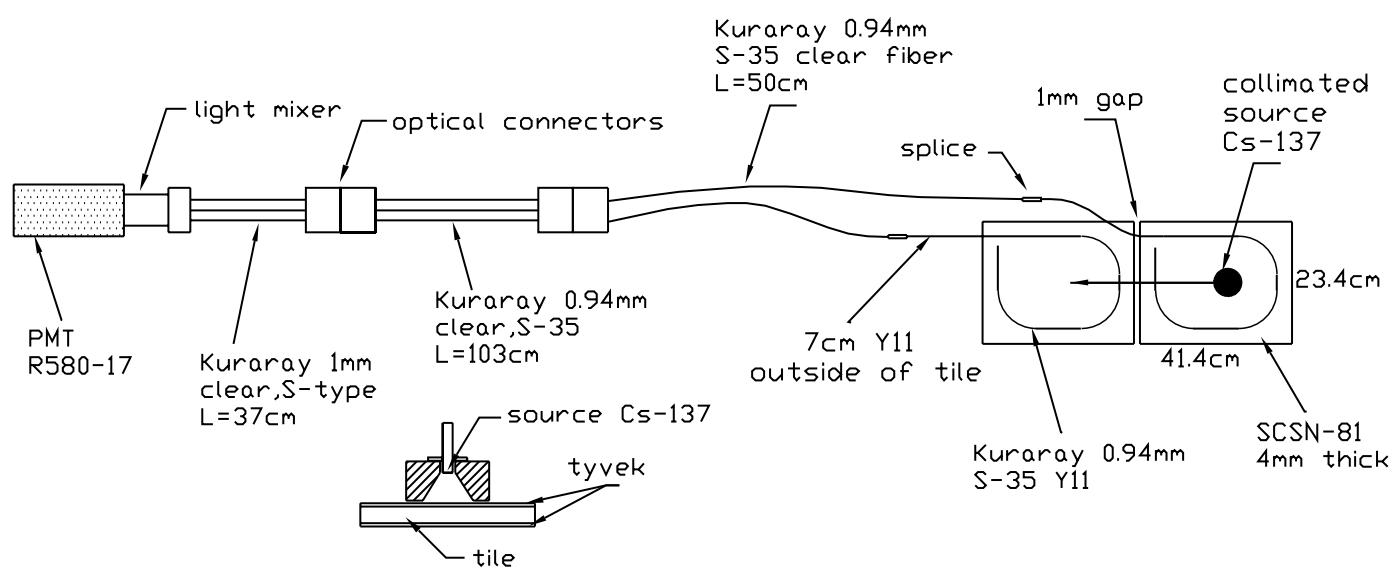

FIGURE 26. Uniformity across a tile using collimated $\gamma$ source. The fiber insertion is parallel to the short side. The measurement is done with both Kuraray and Bicron fibers.
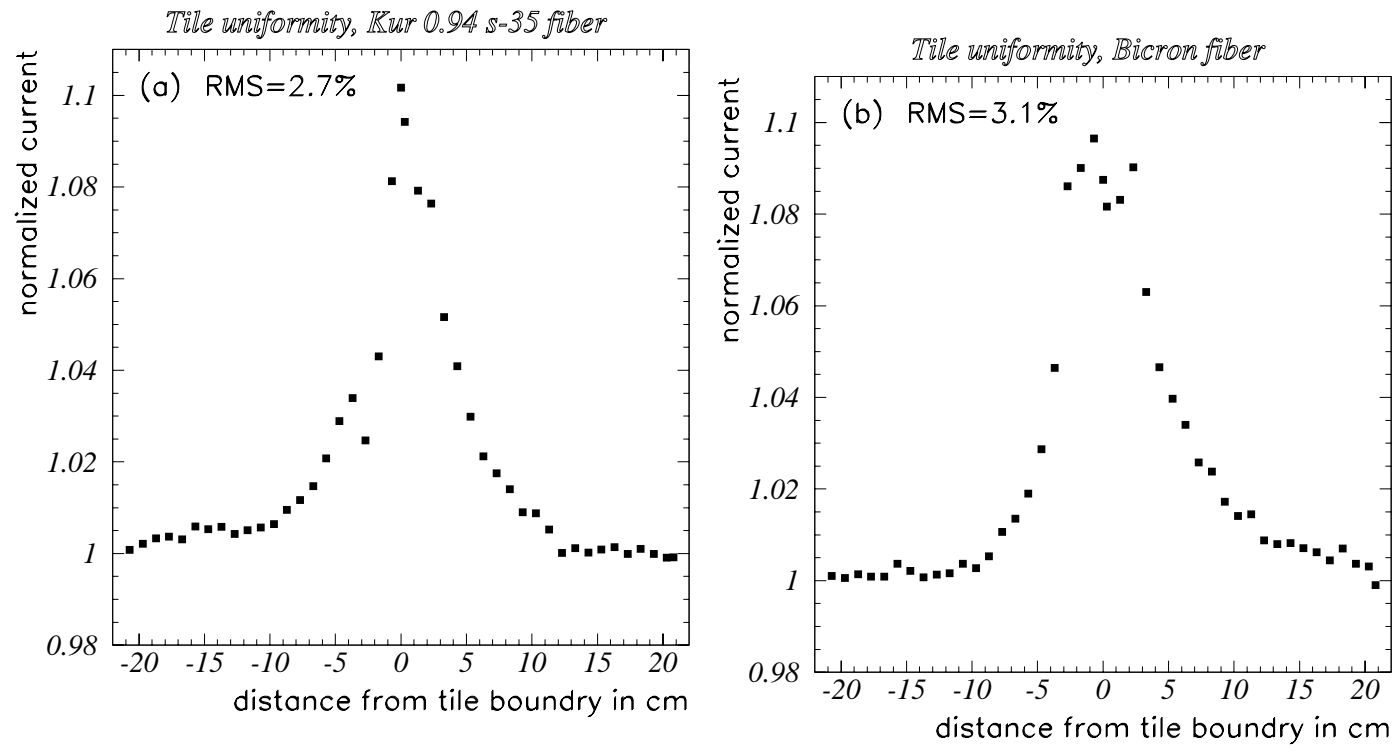

uniformity from affecting the constant term in the resolution.

The HCAL CMS scintillator design has individual tiles glued together with $\mathrm{TiO}_{2}$ loaded epoxy resin [15], to form a "megatile". The configuration at the boundary between tiles is shown in Figure 28. A $0.9 \mathrm{~mm}$ wide "separation groove" is cut to separate 2 tiles, with $1 / 4 \mathrm{~mm}$ of scintillator left uncut on the bottom of the groove. The groove is filled with $\mathrm{TiO}_{2}$ loaded epoxy. The scintillator is marked with a black mark made with the narrow end of a black 
FIGURE 27. Uniformity across a tile using collimated $\gamma$ source. The fiber insertion is parallel to the long side.

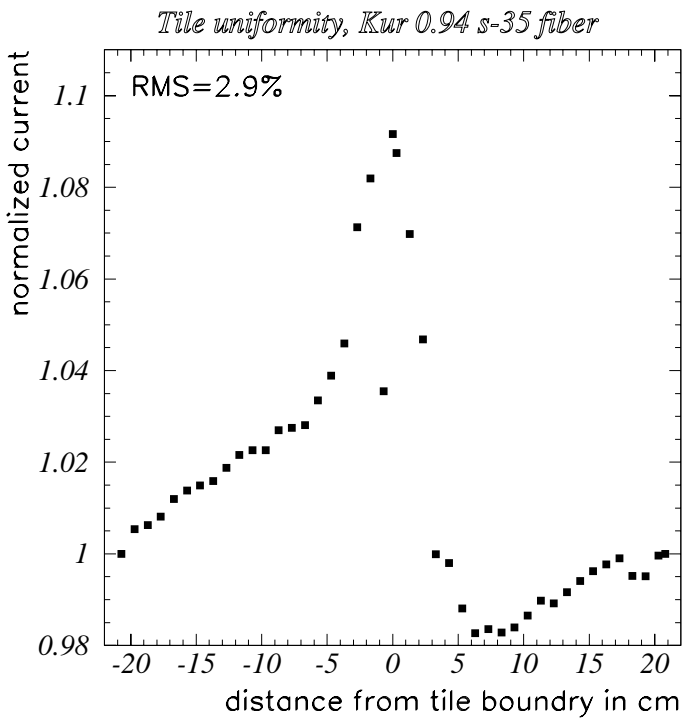

FIGURE 28. The boundary between tiles in CMS HCAL barrel.

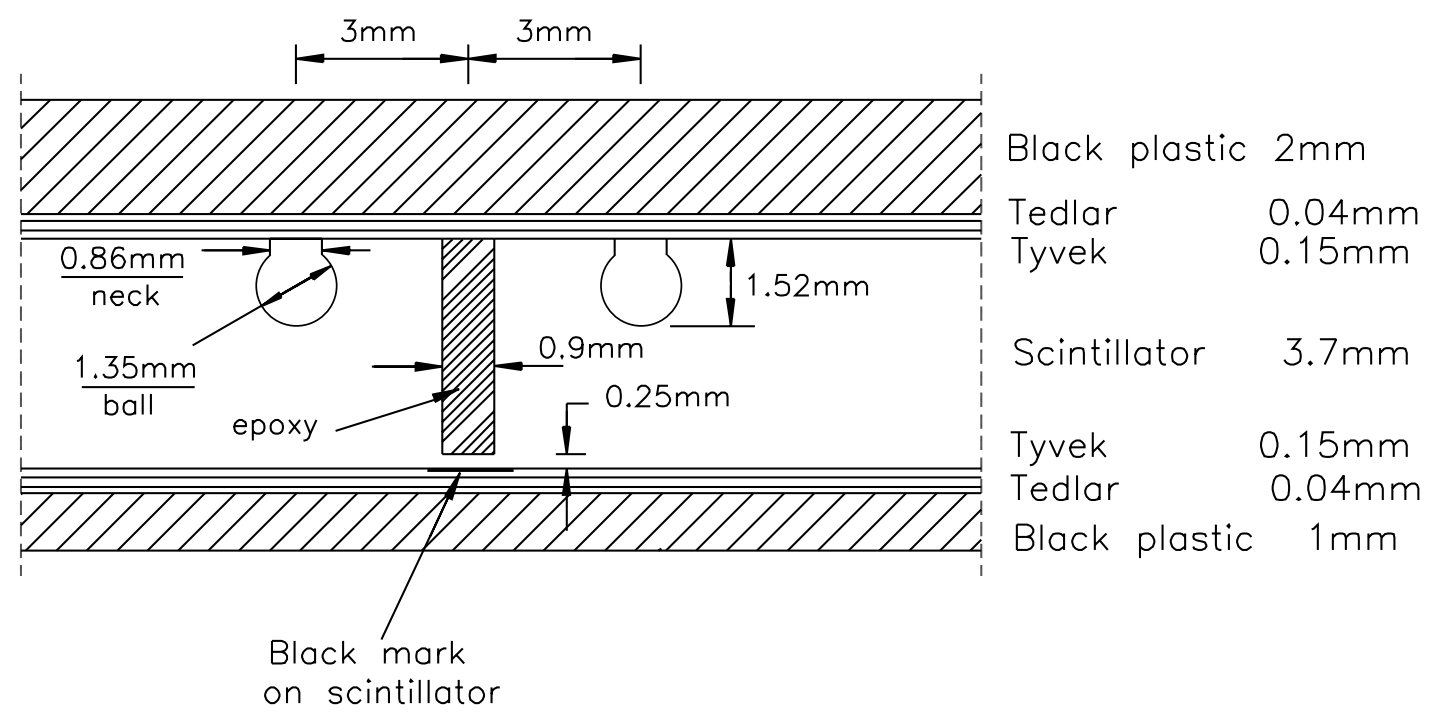

marker pen [16]. The black mark is underneath the separation groove. The black mark is about $1.5 \mathrm{~mm}$ wide, slightly wider than the separation groove. The black mark reduces the light cross talk through the $1 / 4 \mathrm{~mm}$ of scintillator left at the tile boundary.

We constructed a glued megatile consisting of 2 tiles inside a piece of scintillator. The tiles for that glued megatile tile are from tower 10, layer 1. Figure 29 shows the apparatus used to measure the glued megatile. Figure 30 shows 
FIGURE 29. Apparatus to measure transverse tile uniformity with $1 \mathrm{~mm}$ glue joint between the tiles.

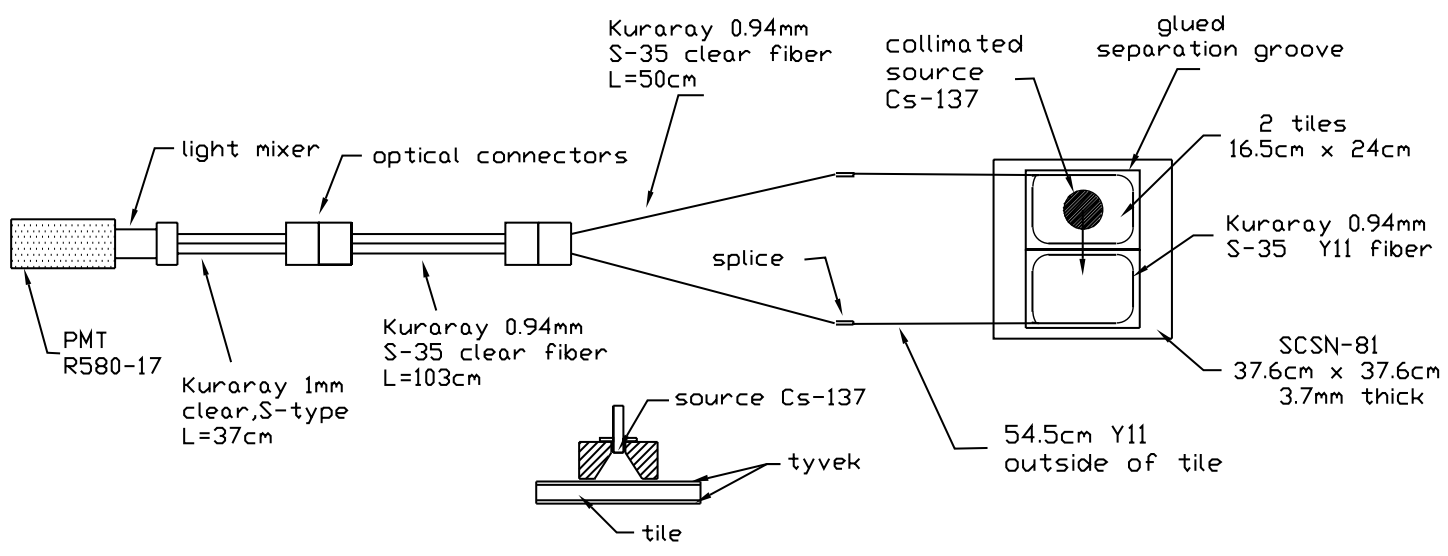

FIGURE 30. Uniformity across the glued megatile using collimated $\gamma$ source.

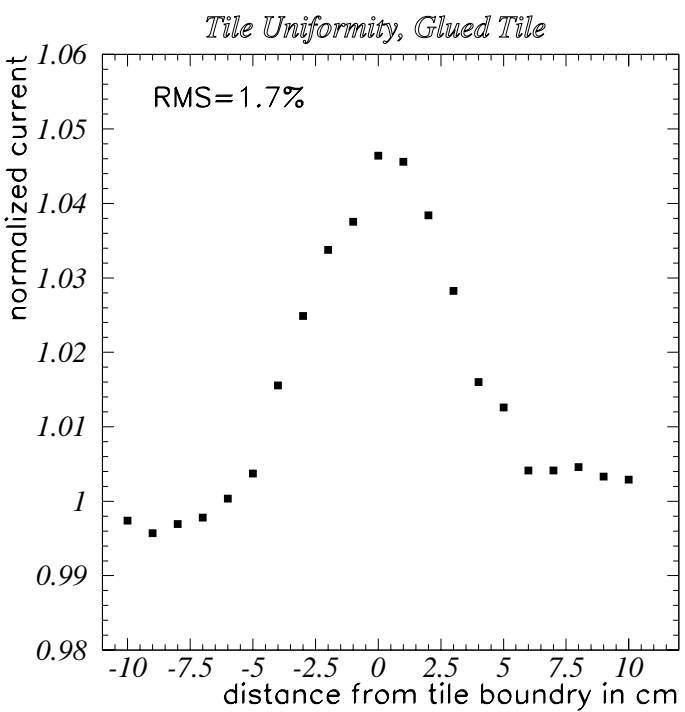

the result. The transverse RMS is roughly $1.7 \%$. The transverse uniformity does not increase the constant term of the resolution.

We have measured the cross talk between the glued tiles. The $1 / 4 \mathrm{~mm}$ of scintillator, left uncut between the two tiles, provides a path for light to pass between 2 tiles. The cross talk is measured by first putting a fiber in one of the tiles. Next, we measure the current with the source on the following three locations: the tile with the fiber, the tile without the fiber, and just off the tile with the fiber. The last location is used to measure the source cross talk. The cross talk is $\sim 1 \%$.

The black mark decreases the light output. We measured the light from glued megatile. Next, the black marks are made on 3 sides of the tile, similar to the way the HCAL CMS barrel tiles will be marked. The light goes down 
roughly $8 \%$.

\section{CONCLUSION}

The CMS R and D enables us to design the optics of HCAL Barrel Calorimeter and predict its performance. We have chosen Kuraray S-35 fiber for the HCAL preproduction prototype because of its excellent flexibility, excellent mirror reflectivity, and high splice transmission. CMS HCAL has chosen to ice polish the fibers, since it enables us to polish many fibers at once. We predict the light of each tile in the barrel using the attenuation lengths of fibers and the absolute light vs the tile size. By varying the position of the splice for each tile, we can optimize the light distribution in a tower. CMS has chosen to have the same length WLS fiber for all layers in a tower. Measurements of the transverse uniformity shows that it does not effect the resolution of the calorimeter.

\section{REFERENCES}

1. P. de Barbaro et al., CDF End Plug Upgrade Calorimeter Design, University of Rochester Preprint UR-1360, Jul. 1994, CDF Note 2545.

2. P. de Barbaro and A. Bodek, Scintillator Tile-Fiber Calorimeters for High Energy Physics: The CDF End Plug Upgrade, Selected Articles, University of Rochester preprint UR 1389, October 1994. References [1], [7], [8], [11], [12]. and [15] are in this document.

3. Kuraray Co., LTD., 8F, Maruzen Building, 3-10, 2-Chome, Nihonbashi, Chuoku, Tokyo, 103-0027, Japan.

4. Bicron Corporation, 12345 Kinsman Road, Newbury, Ohio 44065-9677.

5. DDK Electronics Inc., 3001 Oakmead Village Drive, Santa Clara, Calif. 95051, and DDK Co. Ltd, Yoyogi 2-7-12, Shibuya-ku, Tokyo 151.

6. S. Aota et al., Nucl. Instr. and Meth. A357 (1995) 71.

7. G. Apollinari, P. de Barbaro, and M. Mishina, CDF End Plug Calorimeter Upgrade Project, Proceedings of the IV International Conference on the Calorimetry in High Energy Physics, La Biodola Elba, Sep 19-25, 1993.

8. J.P. Mansour, C. Bromberg, J. Huston, S. Joy, B. Miller, R. Richards, B. Tannenbaum (Michigan State U.), A Semiautomated Splicer for Plastic Optical Fibers, Notre Dame 1993, Proceedings, Scintillating fiber detectors, 534-541.

9. R. Mussa et al., Performance Measurements of Histe-V VLPC Photon Detectors for E835 at FNAL, paper submitted to these proceedings.

10. C. Durandet et al., The Use of WLS Fibers in a Hadronic Calorimeter for the HyperCP Experiment, paper submitted to these proceedings.

11. P. de Barbaro et al., Recent RED Results on Tile/Fiber Calorimeter, University of Rochester preprint UR-1299, SDC-93-407, January 1993. 
12. P. Koehn, Tile/Fiber Results for the Upgraded Plug Hadron Calorimeter, IEEE 1993 Nuclear Science Symposium and Medical Imaging Conference, San Francisco, October 1993.

13. Bicron BC-620 white paint, Bicron Corp, Newbury, OH.

14. P. de Barbaro et al., Performance of a Prototype CMS Hadron Barrel Calorimeter in a Test Beam, Proceedings of the CALOR97, VII International Conference on Calorimetry in High Energy Physics November 9 - 14, 1997 University of Arizona Tucson, Arizona.

15. M. Olsson et al., Techniques for Optical Isolation and Construction of Megatiles, University of Rochester preprint UR-1370.

16. "Doubleshot" ink marker (No. 11120), Pentech International Inc., Edison, NJ. 